\title{
ON SOME GEOMETRIC PROPERTIES OF SLICE REGULAR FUNCTIONS OF A QUATERNION VARIABLE
}

\author{
SORIN G. GAL, J. OSCAR GONZÁLEZ-CERVANTES, AND IRENE SABADINI
}

\begin{abstract}
The goal of this paper is to introduce and study some geometric properties of slice regular functions of quaternion variable like univalence, subordination, starlikeness, convexity and spirallikeness in the unit ball. We prove a number of results, among which an Area-type Theorem, Rogosinski inequality, and a Bieberbach-de Branges Theorem for a subclass of slice regular functions. We also discuss some geometric and algebraic interpretations of our results in terms of maps from $\mathbb{R}^{4}$ to itself. As a tool for subordination we define a suitable notion of composition of slice regular functions which is of independent interest.
\end{abstract}

\section{INTRODUCTION}

The functions we consider in this paper are power series of the quaternion variable $q$ of the form $\sum_{n=0}^{\infty} q^{n} a_{n}$ with quaternionic coefficients $a_{n}$, converging in the unit ball $\mathbb{B}$. These functions are (left) slice regular according to the definition in [20] and also according to the definition in [21]. The two definitions in 20] and in 21] are different, but they give rise to the same class of functions on some particular opens sets called axially symmetric slice domains, that we will introduce in the next section. Slice regular functions are nowadays a widely studied topic, important especially for its application to a functional calculus for quaternionic linear operators, see [12], and to Schur analysis, see [4], and [5] in which Blaschke factors are also studied.

It is then natural to continue the study of this class of functions by considering some geometric properties of slice regular functions like univalence, subordination, starlikeness, convexity and spirallikeness in the unit ball. In the literature, some other geometric properties of this class of functions have been already considered and some results have been proved: the Bloch-Landau theorem, [14, the Bohr theorem, 18, Landau-Toeplitz theorem, [15], (some of these results are collected in [19]) Schwarz-Pick lemma, see [6] and 2] for an alternative, shorter proof. Recently also the BorelCarathéodory theorem has been proved, see [27] and also [3] for a weaker version.

The plan of the paper is as follows. Section 2 contains some known concepts and results useful in the next sections. Section 3 discusses univalence

1991 Mathematics Subject Classification. Primary 30G35; Secondary 30C45.

Key words and phrases. Quaternion, subordination, univalent function, starlike function, convex function, spirallike function, slice regular functions. 
of slice regular functions and several conditions under which a function defined on the open unit ball of the quaternions is univalent. We introduce the counterpart of the Koebe function in the quaternionic setting and obtain an Area-type theorem and a Bieberbach- de Branges result for a subclass of univalent slice regular functions. We also provide some algebraic and geometric interpretation of our result in terms of transformations from $\mathbb{R}^{4}$ to itself. In Section 4 we introduce a notion of composition of formal power series and we study their convergence. We then introduce the notion of subordination of slice regular functions and we prove the Rogosinski inequality. In Section 5 we consider starlike and convex slice regular functions, also discussing some geometric consequences. Finally, in Section 6 , we consider spirallike functions.

\section{Preliminaries}

Let us recall that the quaternion field is defined as

$$
\mathbb{H}=\left\{q=x_{1}+x_{2} i+x_{3} j+x_{4} k ; x_{1}, x_{2}, x_{3}, x_{4} \in \mathbb{R}\right\},
$$

where the imaginary units $i, j, k \notin \mathbb{R}$ satisfy

$$
i^{2}=j^{2}=k^{2}=-1, i j=-j i=k, j k=-k j=i, k i=-i k=j .
$$

It is a noncommutative field and since obviously $\mathbb{C} \subset \mathbb{H}$, it extends the class of complex numbers. On $\mathbb{H}$ can be defined the norm $\|q\|=\sqrt{x_{1}^{2}+x_{2}^{2}+x_{3}^{3}+x_{4}^{2}}$, for $q=x_{1}+x_{2} i+x_{3} j+x_{4} k$.

Let us denote by $\mathbb{S}$ the unit sphere of purely imaginary quaternion, i.e.

$$
\mathbb{S}=\left\{q=i x_{1}+j x_{2}+k x_{3}, \text { such that } x_{1}^{2}+x_{2}^{2}+x_{3}^{3}=1\right\} .
$$

Note that if $I \in \mathbb{S}$, then $I^{2}=-1$. For this reason the elements of $\mathbb{S}$ are also called imaginary units. For any fixed $I \in \mathbb{S}$ we define $\mathbb{C}_{I}:=\{x+I y ; \mid x, y \in$ $\mathbb{R}\}$. It is easy to verify that $\mathbb{C}_{I}$ can be identified with a complex plane, moreover $\mathbb{H}=\bigcup_{I \in \mathbb{S}} \mathbb{C}_{I}$. The real axis belongs to $\mathbb{C}_{I}$ for every $I \in \mathbb{S}$ and thus a real quaternion can be associated to any imaginary unit $I$. Any non real quaternion $q$ is uniquely associated to the element $I_{q} \in \mathbb{S}$ defined by $I_{q}:=\left(i x_{1}+j x_{2}+k x_{3}\right) /\left\|i x_{1}+j x_{2}+k x_{3}\right\|$ and, obviously, $q$ belongs to the complex plane $\mathbb{C}_{I_{q}}$.

Also, recall that for $q \in \mathbb{H} \backslash \mathbb{R}, q=x_{1}+i x_{2}+j x_{3}+k x_{4}$, defining $r:=\|q\|=$ $\sqrt{x_{1}^{2}+x_{2}^{2}+x_{3}^{2}+x_{4}^{2}}$, there exists uniquely $a \in(0, \pi)$ with $\cos (a):=\frac{x_{1}}{r}$ and there exists uniquely $I_{q} \in \mathbb{S}$, such that

$q=r e^{I_{q} a}$, with $I_{q}=i y+j v+k s, y=\frac{x_{2}}{r \sin (a)}, u=\frac{x_{3}}{r \sin (a)}, s=\frac{x_{4}}{r \sin (a)}$.

Now, if $q \in \mathbb{R}$, then we choose $a=0$, if $q>0$ and $a=\pi$ if $q<0$, and as $I_{q}$ we choose an arbitrary fixed $I \in \mathbb{S}$. So that if $q \in \mathbb{R} \backslash\{0\}$, then again we can write $q=\|q\|(\cos (a)+I \sin (a))$ (but with non-unique $I$ ).

The above is called the trigonometric form of the quaternion number $q \neq 0$ and $a$ denoted by $\arg (q)$ is called the argument of the quaternion $q$. 
Evidently, "a" could be considered as the angle between the real axis and the segment $[0, q]$ in $\mathbb{R}^{4}$ (or, in other words, the angle between the real axis and the radius in $\mathbb{R}^{4}$ passing through origin and the geometric image in $\mathbb{R}^{4}$ of $q$ ).

If $q=0$, then we do not have a trigonometric form for $q$ (exactly as in the complex case).

For our purposes we will need the following concept of analyticity of functions of a quaternion variable.

Definition 2.1. Let $U$ be an open set in $\mathbb{H}$ and $f: U \rightarrow \mathbb{H}$ real differentiable. $f$ is called left slice regular or slice hyperholomorphic if for every $I \in \mathbb{S}$, its restriction $f_{I}$ to the complex plane $\mathbb{C}_{I}=\mathbb{R}+I \mathbb{R}$ passing through origin and containing $I$ and 1 satisfies

$$
\bar{\partial}_{I} f(x+I y):=\frac{1}{2}\left(\frac{\partial}{\partial x}+I \frac{\partial}{\partial y}\right) f_{I}(x+I y)=0,
$$

on $U \cap \mathbb{C}_{I}$. The class of slice regular functions on $U$ will be denoted by $\mathcal{R}(U)$.

Let $f \in \mathcal{R}(U)$. The so called left (slice) $I$-derivative of $f$ at a point $q=x+I y$ is given by

$$
\partial_{I} f_{I}(x+I y):=\frac{1}{2}\left(\frac{\partial}{\partial x} f_{I}(x+I y)-I \frac{\partial}{\partial y} f_{I}(x+I y)\right) .
$$

Analogously, a function is called right slice regular if

$$
\left(f_{I} \bar{\partial}_{I}\right)(x+I y):=\frac{1}{2}\left(\frac{\partial}{\partial x} f_{I}(x+I y)+\frac{\partial}{\partial y} f_{I}(x+I y) I\right)=0,
$$

on $U \cap \mathbb{C}_{I}$.

In this case, the right $I$-derivative of $f$ at $q=x+I y$ is given by

$$
\partial_{I} f_{I}(x+I y):=\frac{1}{2}\left(\frac{\partial}{\partial x} f_{I}(x+I y)-\frac{\partial}{\partial y} f_{I}(x+I y) I\right) .
$$

Let us now introduce a suitable notion of derivative:

Definition 2.2. Let $U$ be an open set in $\mathbb{H}$, and let $f: U \rightarrow \mathbb{H}$ be a slice regular function. The slice derivative of $f, \partial_{s} f$, is defined by:

$$
\partial_{s}(f)(q)= \begin{cases}\partial_{I}(f)(q) & \text { if } q=x+I y, \quad y \neq 0, \\ \frac{\partial f}{\partial x}(x) & \text { if } q=x \in \mathbb{R} .\end{cases}
$$

The definition of slice derivative is well posed because it is applied only to slice regular functions and thus

$$
\frac{\partial}{\partial x} f(x+I y)=-I \frac{\partial}{\partial y} f(x+I y) \quad \forall I \in \mathbb{S},
$$

and therefore, analogously to what happens in the complex case,

$$
\partial_{s}(f)(x+I y)=\partial_{I}(f)(x+I y)=\partial_{x}(f)(x+I y) .
$$


We will often write $f^{\prime}(q)$ instead of $\partial_{s} f(q)$.

If $f$ is a slice regular function, then also its slice derivative is slice regular, in fact

and therefore

$$
\bar{\partial}_{I}\left(\partial_{s} f(x+I y)\right)=\partial_{s}\left(\bar{\partial}_{I} f(x+I y)\right)=0,
$$

$$
\partial_{s}^{n} f(x+I y)=\frac{\partial^{n} f}{\partial x^{n}}(x+I y) .
$$

Among the useful tools from the general theory on slice regular functions, we recall some useful facts, collected in a following theorem. For the definition of axially symmetric s-domain we refer the reader e.g. to [12. For our purposes, it is enough to know that balls in $\mathbb{H}$ are examples of axially symmetric s-domains.

Theorem 2.3. Let $U \subseteq \mathbb{H}$ be an axially symmetric s-domain and $f: U \rightarrow \mathbb{H}$ be a (left) slice regular function.

(1) (Representation Formula) The following equality holds for all $q=$ $x+I y \in \Omega$ :

$$
f(x+I y)=\frac{1}{2}[f(x+J y)+f(x-J y)]+\frac{1}{2} I J[f(x-J y)-f(x+J y)] .
$$

(2) (General Representation Formula) The following equality holds for all $q=x+I y \in \Omega:$

$f(x+I y)=(J-K)^{-1}[J f(x+J y)-K f(x+K y)]+I(J-K)^{-1}[f(x+J y)-f(x+K y)]$.

(3) (Splitting Lemma, see. e.g. Lemma 4.1.7, p. 117 in [12]) For every $I \in \mathbb{S}$ and every $J \in \mathbb{S}$, perpendicular to $I$, there are two holomorphic functions $F, G: U \cap \mathbb{C}_{I} \rightarrow \mathbb{C}_{I}$, such that for any $z=x+$ Iy we have $f_{I}(z)=F(z)+G(z) J$.

(4) (see e.g. Corollary 4.3.6, p. 121 in [12]) For all $x, y \in \mathbb{R}$ such that $x+I y \in U$, there exist $a, b \in \mathbb{H}$ such that $f(x+I y)=\alpha(x, y)+$ $I \beta(x, y)$, for all $I \in \mathbb{S}$.

(5) (see e.g. Corollary 4.3.4, p. 121, [12]) Define $D \subset \mathbb{R}^{2}$ such that $(x, y) \in D$ implies $x+I y \in U$. The function $f$ is slice regular if and only if there exist two differentiable functions $\alpha, \beta: D \subset \mathbb{R}^{2} \rightarrow \mathbb{H}$ satisfying on $D, \alpha(x, y)=\alpha(x,-y), \beta(x, y)=-\beta(x,-y)$ and the Cauchy-Riemann system $\frac{\partial \alpha}{\partial x}-\frac{\partial \beta}{\partial y}=0, \frac{\partial \beta}{\partial x}+\frac{\partial \alpha}{\partial y}=0$, such that $f(x+I y)=\alpha(x, y)+I \beta(x, y)$. The functions $\alpha(x, y)$ and $\beta(x, y)$ do not depend on $I \in \mathbb{S}$.

(6) (See e.g. Theorem 2.7 in [20]) Let $B(0 ; R)=\{q \in \mathbb{H} ;\|q\|<R\}$. A function $f: B(0 ; R) \rightarrow \mathbb{H}$ is (left) slice regular on $B(0 ; R)$ if and only if it has a series representation of the form

$$
f(q)=\sum_{n=0}^{\infty} q^{n} \frac{1}{n !} \cdot \frac{\partial^{n} f}{\partial x^{n}}(0)
$$

uniformly convergent on $B(0 ; R)$. 
A useful subclass of slice regular functions is denoted by the letter $\mathcal{N}$ (see e.g. [12], p. 152, Definition 4.11.2) and it can be characterized in various ways. Let $U$ be an open set in $\mathbb{H}$ and define

$$
\mathcal{N}(U)=\left\{f \text { slice regular in } U: f\left(U \cap \mathbb{C}_{I}\right) \subseteq \mathbb{C}_{I}, \quad \forall I \in \mathbb{S}\right\} .
$$

It is possible to prove that if $U$ is an axially symmetric s-domain, then $f \in \mathcal{N}(U)$ if and only if it is of the form described in Theorem 2.3 (3) where $\alpha, \beta$ are real valued.

Let us consider the ball $B(0 ; R)$ with center at the origin and radius $R>0$; it is immediate that a function slice regular on $B(0 ; R)$ belong to $\mathcal{N}$ if and only if its power series expansion has real coefficients. Thus the class $\mathcal{N}$ includes all elementary transcendental functions, like exponential, logarithm, sine, cosine, etc.

Finally, if we denote by $Z_{\mathbb{H}}$ the conjugate quaternion, that is $Z_{\mathbb{H}}(q)=\bar{q}$, it can be shown, see [10], that a function $f \in \mathcal{N}(U)$, where $U$ is an axially symmetric s-domain, if and only is it satisfies $f=Z_{\mathbb{H}} \circ f \circ Z_{\mathbb{H}}$. This property is called in [10] C-property, where "C" stands for conjugation. In analogy to the terminology in the complex case, where functions satisfying $f(\bar{z})=$ $\overline{f(z)}$ or, equivalently, $f(z)=\overline{f(\bar{z})}$ are called intrinsic, we will call these functions quaternionic intrinsic. Quaternionic intrinsic functions are the building blocks of slice regular functions in the sense of the following result:

Proposition 2.4. (See Proposition 3.12 in [10]) Let $U$ be an axially symmetric s-domain and let $\{1, \mathbf{i}, \mathbf{j}, \mathbf{i j}\}$ be a basis of $\mathbb{H}$, as a real vector space. Then the right vector space $\mathcal{R}(U)$ of slice regular functions on $U$ can be decomposed as:

$$
\mathcal{R}(U)=\mathcal{N}(U) \oplus \mathcal{N}(U) \mathbf{i} \oplus \mathcal{N}(U) \mathbf{j} \oplus \mathcal{N}(U) \mathbf{i j} .
$$

Functions belonging to the class $\mathcal{N}$ have nice properties, see for example 8] for applications to the spectral mapping theorem. It is also important to mention that, in general, the composition of two slice regular functions is not slice regular. However we have

Theorem 2.5. Let $f \in \mathcal{R}(U)$ and $g \in \mathcal{N}(V)$ be such that $g(V) \subseteq U$. Then $f \circ g \in \mathcal{R}(V)$.

\section{Univalence of Slice Regular Functions}

Definition 3.1. Let $f \in \mathcal{R}(B(0 ; 1))$, where $B(0 ; 1)=\{q \in \mathbb{H} ;\|q\|<1\}$, then :

(1) $f$ is called univalent in $B(0 ; 1)$ if it is injective in $B(0 ; 1)$;

(2) For $I \in \mathbb{S}, f$ is $I$-univalent if $\left.f\right|_{\mathbb{D}_{I}}$ is univalent, where $\mathbb{D}_{I}=B(0 ; 1) \cap$ $\mathbb{C}_{I}$.

Remark 3.2. 
(1) From the definition it is clear that if $f \in \mathcal{R}(B(0 ; 1))$ is univalent then $\mathrm{f}$ is $I$-univalent for any $I \in \mathcal{S}$.

Consider the slice regular function

$$
f(q)=q^{2}+q J, \quad q \in B(0 ; 1),
$$

where $J \in \mathbb{S}$ is a fixed element. Then $f$ is $I$-univalent for any $I \in$ $\mathbb{S} \backslash\{J\}$ and for $z \in \mathbb{D}_{J}$ the restriction

$$
\left.f\right|_{\mathbb{D}_{J}}(z)=z^{2}+z J, \quad z \in \mathbb{D}_{J}
$$

is not univalent. We conclude that $f$ is not $J$-univalent and therefore is not univalent in $B(0 ; 1)$.

(2) Consider $f \in \mathcal{N}(B(0 ; 1))$ and suppose $I \in \mathbb{S}$ such that $f$ is an $I$ univalent function. As $f$ can be expressed by $f(q)=\alpha(x, y)+$ $I \beta(x, y)$, for all $q=x+I y \in \mathbb{B}(0 ; 1)$, where $\alpha$ and $\beta$ are real valued functions, then $\alpha$ and $\beta$ satisfy the condition : if $x_{i}^{2}+y_{i}^{2}<1, i=1,2$ and $\left(x_{1}, y_{1}\right) \neq\left(x_{2}, y_{2}\right)$ then $\alpha\left(x_{1}, y_{1}\right) \neq \alpha\left(x_{2}, y_{2}\right)$ or $\beta\left(x_{1}, y_{1}\right) \neq$ $\beta\left(x_{2}, y_{2}\right)$. This clearly implies that $f$ is $J$-univalent for all $J \in \mathbb{S}$. Finally, if $w, q$ belong to different slices then $f(w) \neq f(q)$. Therefore $f$ is univalent too. For example, the functions

$$
f(q)=q(1-q)^{-1}, \quad g(q)=q\left(1-q^{2}\right)^{-1}, \quad h(q)=q-\frac{1}{2} q^{2}, \quad q \in \mathbb{B}(0 ; 1)
$$

are univalent.

Suppose that $f: B(0 ; 1) \rightarrow \mathbb{H}$ is a slice (left) regular function, i.e. $f(q)=\sum_{k=0}^{\infty} q^{k} a_{k}$, for all $q \in B(0 ; 1)$. A natural question is if the condition $\operatorname{Re}\left[\partial_{s}(f)(q)\right]>0$, for all $q \in B(0 ; 1)$ implies that $f$ is univalent (injective) on $B(0 ; 1)$, as it happens in the complex variable case.

Partial answers to this question are the following results.

Theorem 3.3. Let $f: B(0 ; 1) \rightarrow \mathbb{H}$ be a slice (left) regular function, i.e. $f(q)=\sum_{k=0}^{\infty} q^{k} a_{k}$, for all $q \in B(0 ; 1)$. Suppose also that $\operatorname{Re}\left[\partial_{s}(f)(q)\right]>0$ for all $q \in B(0 ; 1)$. Then one has the following properties

(1) For any $I \in \mathbb{S}, f$ is univalent on $B(0 ; 1) \cap \mathbb{C}_{I}$.

(2) Suppose that $a_{0}=0$ and $a_{1}=1$, then $\frac{1-\|q\|}{1+\|q\|} \leq \operatorname{Re}\left[\partial_{s} f(q)\right] \leq$ $\left\|\partial_{s} f(q)\right\| \leq \frac{1+\|q\|}{1-\|q\|}, \quad q \in B(0 ; 1)$.

If the first or the third inequality becomes in an equality for some $q \neq 0$ then there exist $I \in \mathbb{S}$ and $\theta \in \mathbb{R}$ such that

$$
f(q)=q+\sum_{n=2}^{\infty} q^{n} \frac{2 e^{I(n-1) \theta}}{n}, \quad q \in B(0 ; 1) .
$$


Moreover, $\left\|n a_{n}\right\| \leq 2$ for each $n \in \mathbb{N}$. But if for some $n_{0} \in \mathbb{N}$ one has that $\left\|a_{n_{0}}\right\|=\frac{2}{n_{0}}$ if and only if

$$
a_{k n_{0}+1}=\frac{2}{k n_{0}+1}\left(\frac{n_{0} a_{n_{0}}}{2}\right)^{k}
$$

for each $k \in \mathbb{N}$. Note that if $\left\|a_{2}\right\|=1$ then there exist $I \in \mathbb{S}$ and $\theta \in \mathbb{R}$ such that

$$
f(q)=q+\sum_{n=2}^{\infty} q^{n} \frac{2 e^{I(n-1) \theta}}{n}, \quad q \in B(0 ; 1) .
$$

Proof. (1) Let $z_{1}, z_{2} \in \mathbb{H}$ be with $z_{1} \neq z_{2}$. Denoting $z(t)=t z_{2}+(1-t) z_{1}$, $t \in[0,1]$, we have

$\frac{d\left[(z(t))^{k}\right]}{d t}=\sum_{p=0}^{k-1} z(t)^{p} \cdot z_{0} \cdot z(t)^{k-1-p}$, where $z_{0}=z_{2}-z_{1}, k \geq 1$.

Let $z_{1}, z_{2} \in B(0 ; 1) \bigcap \mathbb{C}_{I}$ be with an arbitrary $I \in \mathbb{S}, z_{1} \neq z_{2}$. Since obviously $z_{0} \in \mathbb{C}_{I}$ and $z(t) \in \mathbb{C}_{I}$ for all $t \in[0,1]$, due to the commutativity (in $\mathbb{C}_{I}$ ) we easily get $\frac{d\left[(z(t))^{k}\right]}{d t}=z_{0} \cdot k z(t)^{k-1}$ which (due to the uniform convergence of the series) immediately implies that

$$
\frac{d[f(z(t))]}{d t}=\sum_{k=0}^{\infty} \frac{d\left[(z(t))^{k}\right]}{d t} a_{k}=z_{0} \cdot \sum_{k=1}^{\infty} k z(t)^{k-1} a_{k}=z_{0} \cdot \partial_{s}(f)(z(t)) .
$$

Reasoning now as in the complex variable case, we get

$$
f\left(z_{2}\right)-f\left(z_{1}\right)=\int_{0}^{1} \frac{d[f(z(t))]}{d t} d t=z_{0} \int_{0}^{1} \partial_{s}(f)(z(t)) d t .
$$

Since $z_{0} \neq 0$, this implies $z_{0}^{-1} \cdot\left[f\left(z_{2}\right)-f\left(z_{1}\right)\right]=\int_{0}^{1} \partial_{s}(f)(z(t)) d t$ and

$$
\operatorname{Re}\left[z_{0}^{-1} \cdot\left[f\left(z_{2}\right)-f\left(z_{1}\right)\right]=\operatorname{Re}\left[\int_{0}^{1} \partial_{s}(f)(z(t)) d t\right]>0,\right.
$$

that is $f\left(z_{2}\right)-f\left(z_{1}\right) \neq 0$.

(2) From the Carathéodory theorem for slice regular functions, see [27, Theorem 3], $0<\operatorname{Re} \partial_{s} f(q)$ for each $q \in B(0 ; 1)$ implies

$$
\frac{1-\|q\|}{1+\|q\|} \leq \operatorname{Re} \partial_{s} f(q) \leq\left\|\partial_{s} f(q)\right\| \leq \frac{1+\|q\|}{1-\|q\|}, \quad q \in B(0 ; 1),
$$

and if the first or the third inequality becomes in an equality for some $q \neq 0$ then

$$
\partial_{s} f(q)=\left(1-q e^{I \theta}\right)^{-*} *\left(1+q e^{I \theta}\right), \quad q \in B(0 ; 1),
$$

for some $I \in \mathbb{S}$ and $\theta \in \mathbb{R}$. In particular, if $q \in \mathbb{D}_{I}$ one obtains

$$
\partial_{s} f(q)=\left(1+q e^{I \theta}\right) \frac{1}{1-q e^{I \theta}}=\left(1+q e^{I \theta}\right) \sum_{n=0}^{\infty} q^{n} e^{I n \theta},
$$




$$
\begin{gathered}
=\sum_{n=0}^{\infty} q^{n} e^{I n \theta}+\sum_{n=0}^{\infty} q^{n+1} e^{I(n+1) \theta}=1+\sum_{n=1}^{\infty} q^{n} e^{I n \theta}+\sum_{n=1}^{\infty} q^{n} e^{I n \theta}, \\
=1+2 \sum_{n=1}^{\infty} q^{n} e^{I n \theta}, \quad q \in B(0 ; 1) .
\end{gathered}
$$

By the uniqueness of the slice regular extension, the equality $\partial_{s} f(q)=$

$$
\begin{gathered}
1+2 \sum_{n=1}^{\infty} q^{n} e^{I n \theta} \text { holds on } B(0 ; 1) \text {. Thus, if } f(q)=q+\sum_{n=2}^{\infty} q^{n} a_{n} \text { then } \\
1+\sum_{n=2}^{\infty} q^{n-1} n a_{n}=\partial_{s} f(q)=1+2 \sum_{n=1}^{\infty} q^{n} e^{I n \theta} \\
1+\sum_{n=1}^{\infty} q^{n}(n+1) a_{(n+1)}=\partial_{s} f(q)=1+2 \sum_{n=1}^{\infty} q^{n} e^{I n \theta} .
\end{gathered}
$$

Therefore $a_{n+1}=\frac{2 e^{\operatorname{In} \theta}}{n+1}$ for each $n \in \mathbb{N}$, or equivalently $a_{n}=$ $\frac{2 e^{I(n-1) \theta}}{n}$ for each $n=2,3,4, \ldots$.

(3) The proof is a direct application to the Carathéodory theorem for slice regular functions to $\partial_{s} f$ and it follows with computations very similar to those in the previous case.

In the special case of intrinsic functions, we have:

Theorem 3.4. Let $f \in \mathcal{N}(B(0 ; 1))$ and suppose that there exists $I \in \mathbb{S}$ with $\operatorname{Re}\left[\partial_{s}(f)(q)\right]>0$ for all $q \in \mathbb{D}_{I}$. Then $\operatorname{Re}\left[\partial_{s}(f)(q)\right]>0$ for all $q \in B(0 ; 1)$ and $f$ is univalent in $B(0 ; 1)$.

Proof. From the result in the classical complex case we have that $f$ is $I$ univalent. The two statement follow from Remark 3.2, point 2 and previous theorem. Since for all $I \in \mathbb{S}$ one has $\operatorname{Re}\left[\partial_{s} f(x+y I)\right]=\partial_{x} \alpha(x, y)>0$ for all $x^{2}+y^{2}<1$.

Example 1. By the above Theorem 3.4, it follows that $f(q)=-2+2 \sum_{k=1}^{\infty} \frac{q^{k}}{k}$ is an univalent function in $B(0 ; 1)$. Indeed, it is known that $f(z)=-2-$ $2 \log (1-z)$ is analytic in the open unit disk $\mathbb{D}_{1}$ and $\operatorname{Re}\left[f^{\prime}(z)\right]>0$, for all $z \in \mathbb{D}_{1}$, therefore it is univalent in $\mathbb{D}_{1}$. Also, since $\log (1-z)=-\sum_{k=1}^{\infty} \frac{z^{k}}{k}$, we get $f(z)=-2+2 \sum_{k=1}^{\infty} \frac{z^{k}}{k}$, for all $z \in \mathbb{D}_{1}$.

Remark 3.5. Although the above results are very simple, they seem to be very useful to produce easily many examples of injective functions in $B(0 ; 1)$. It is clear that an attempt to prove directly the injectivity on $B(0 ; 1)$ of $f$ in the above example seems to be a difficult task. 
In the sequel we will consider the function

$$
K(q)=q \cdot\left[(1-q)^{2}\right]^{-1}
$$

that corresponds to the quaternionic Koebe function. It is immediate that $K(q)=\sum_{n=1}^{\infty} n q^{n}$ since the equality holds for the restriction to a complex plane $\mathbb{C}_{I}$ and $K(q)$ is slice regular and extends (uniquely) $K(z), z \in \mathbb{C}_{I}$.

Example 2. Let $f(q)=q+\sum_{k=2}^{\infty} q^{k} a_{k}$ be a slice regular function on $B(0 ; 1)$, normalized, i.e. $f(0)=1-\partial_{s}(f)(0)=0$. If $\varphi \in \mathbb{R}$ and $I \in \mathbb{S}$, then $R_{\varphi, I}(q)=e^{-I \varphi} \cdot f\left(e^{I \varphi} \cdot q\right)=q\left[1+\sum_{k=1}^{\infty}\left(e^{I \varphi} \cdot q\right)^{k} a_{k+1}\right]$ is called a rotation of $f$. A simple reasoning shows that if $f$ is univalent on $B(0 ; 1)$ then for any $\varphi \in \mathbb{R}, I \in \mathbb{S}, r \in(0,1)$, so is $R_{\varphi, I}$. As an application, the rotation of the quaternionic Koebe function $K(q)=q \cdot\left[(1-q)^{2}\right]^{-1}$,

$$
K_{\varphi, I}(q)=e^{-I \varphi} \cdot K\left(e^{I \varphi} \cdot q\right)=q\left[1+\sum_{k=2}^{\infty} k\left(e^{I \varphi} \cdot q\right)^{k-1}\right],
$$

is univalent as function of $q$ (but not regular) on the ball $B(0 ; 1)$, because according to Theorem 3.4, the Koebe function $f(q)=q+2 q^{2}+3 q^{3}+\ldots+$ is univalent on $B(0 ; 1)$ (see also Section 5 , before Remark 5.15). Note that here for all $a, b \in \mathbb{H}$ we have $(a \cdot b)^{k}=(a \cdot b) \cdot(a \cdot b) \cdot \ldots \cdot(a \cdot b)$ of $k$-times.

Remark 3.6. From $f \in \mathcal{N}(B(0 ; 1))$ it is clear that in Theorem 3.4 we have $\partial_{s}(f)(q)=f^{\prime}(q), q \in B(0 ; 1)$ and that $f^{\prime} \in \mathcal{N}(B(0 ; 1))$. Considering now $f$ and $f^{\prime}$ on a slice $\mathbb{D}_{I}:=B(0 ; 1) \cap \mathbb{C}_{I}$, from the complex case (see e.g. [26], p. 78), the condition $\operatorname{Re}\left[\partial_{s}(f)(q)\right]>0$ for all $q \in \mathbb{D}_{I}$ implies that $\left|\arg \left[\left.f^{\prime}\right|_{I}(q)\right]\right|<\frac{\pi}{2}$, for all $q \in \mathbb{D}_{I}$. But by the definition of argument, see the Introduction, it is evident that $\arg \left[\left.f^{\prime}\right|_{I}(q)\right]$ does not depend on $I \in \mathbb{S}$, which finally leads to the following geometric interpretation of the condition $\operatorname{Re}\left[\partial_{s}(f)(q)\right]>0, q \in B(0 ; 1)$ in Theorem 3.4 .

$$
\left|\arg \left[f^{\prime}(q)\right]\right|<\frac{\pi}{2}, \text { for all } q \in B(0 ; 1) .
$$

As in the complex case, a function satisfying the above inequality can be called of bounded rotation.

Definition 3.7. Let $I \in \mathbb{S}$ be a fixed element. We define the following sets

$$
\mathcal{S}=\left\{f \in \mathcal{R}(B(0 ; 1)) \mid f \text { is univalent in } B(0 ; 1), f(0)=0, \partial_{s} f(0)=1\right\}
$$

and

$\mathcal{S}_{I}=\left\{f \in \mathcal{R}(B(0 ; 1)) \mid f\right.$ is $I$-univalent in $\left.B(0 ; 1), f(0)=0, \partial_{s} f(0)=1\right\}$.

Note that $\mathcal{S} \subset \mathcal{S}_{I}$ for each $I \in \mathbb{S}$. The converse does not hold, as shown in Remark 3.2 .

As in the classical complex case, there exist some operators which preserve the set $\mathcal{S}$ as illustrated in the next result.

Theorem 3.8. (1) If $0<r<1$, then $f \in \mathcal{S}$ if and only if $r^{-1} f(r q) \in \mathcal{S}$. 
(2) Let $u \in \mathbb{H}$ be such that $|u|=1$, let $f \in \mathcal{S}$ and $g(q)=u f(\bar{u} q u) \bar{u}$. Then $g \in \mathcal{S}$.

(3) Let $f \in \mathcal{S} \cap \mathcal{N}(B(0 ; 1))$ and $g \in \mathcal{R}(f(B(0 ; 1)))$ an univalent function with $g(0)=0$ and $\partial_{s} g(0)=1$. Then $g \circ f \in \mathcal{S}$.

(4) Let $f \in \mathcal{S} \cap \mathcal{N}(B(0,1))$ and $a \in \mathbb{R} \backslash(f(B(0 ; 1)) \cap \mathbb{R})$. Then the function $g(q)=f(q) a *(a-f(q))^{-*}, \quad q \in B(0 ; 1)$, belongs to $\mathcal{S} \cap$ $\mathcal{N}(B(0,1))$.

(5) Let $f \in \mathcal{S} \cap \mathcal{N}(B(0,1))$, then $g(q)=\sqrt{f\left(q^{2}\right)}$, belongs to $\mathcal{S} \cap \mathcal{N}(B(0,1))$.

Proof.

(1) The assertion immediately follows with direct computations. The function $r^{-1} f(r q)$ is called a dilation of $f$.

(2) Consider $f(q)=\sum_{n=0}^{\infty} q^{n} a_{n}$ where $q \in B(0 ; 1)$ and $\left(a_{n}\right) \subset \mathbb{H}$. Thus from the uniform convergence one has

$$
g(q)=u f(\bar{u} q u) \bar{u}=u \sum_{n=0}^{\infty}(\bar{u} q u)^{n} a_{n} \bar{u}=\sum_{n=0}^{\infty} q^{n} u a_{n} \bar{u}, \quad q \in B(0 ; 1)
$$

and so $g \in \mathcal{R}(B(0 ; 1))$. Since $f \in \mathcal{S}$ we have $a_{0}=0, a_{1}=1$ and so $u a_{0} \bar{u}=0$ and $u a_{1} \bar{u}=1$. Let $q, r \in B(0 ; 1)$ be such that $g(q)=g(r)$. Then $u f(\bar{u} q u) \bar{u}=u f(\bar{u} r u) \bar{u}$ or, equivalently, $f(\bar{u} q u)=f(\bar{u} r u)$. As $f \in \mathcal{S}$ one concludes that $q=r$, thus $g \in \mathcal{S}$.

(3) As $f \in \mathcal{N}(B(0 ; 1))$ then $g \circ f \in \mathcal{R}(B(0 ; 1))$ and note that $\partial_{s}(g \circ$ $f)(0)=\partial_{s} g(f(0)) \partial_{s} f(0)$. The univalence of $g \circ f$ is immediate.

(4) From the usual complex case, see [16], p. 27, point (vi), one has that $g \in \mathcal{S}_{I} \cap \mathcal{N}(B(0,1))$ for each $I \in \mathbb{S}$ and one concludes using Remark 3.2, point 2 .

(5) Let us write $f(q)=q+a_{2} q^{2}+a_{3} q^{3}+\ldots$. Since $f$ vanishes just at the origin and reasoning on a fixed slice $\mathbb{C}_{I}$ as in the complex case in [16], pp.27-28, we can choose the single-valued branch of the square root given by

$$
g(q)=q\left(1+a_{2} q^{2}+a_{3} q^{4}+\ldots\right)^{1 / 2}=q+b_{3} q^{3}+b_{5} q^{5}+\ldots
$$

The fact that $g$ is univalent in $B(0 ; 1) \cap \mathbb{C}_{I}$ can be proved as in the complex case, see [16], p. 28. Namely, if $g\left(q_{1}\right)=g\left(q_{2}\right)$ with $q_{1} \neq q_{2}$ then $f\left(q_{1}^{2}\right)=f\left(q_{2}^{2}\right)$ but since $f$ is univalent this implies $q_{1}=-q_{2}$ and so $g\left(q_{1}\right)=-g\left(q_{2}\right)$ since $g$ is an odd function. Finally, the univalence of $g \in \mathcal{N}(B(0,1))$ on $B(0 ; 1)$ follows from Remark 3.2, point 2 .

A result ensuring univalence of a function is the following:

Theorem 3.9. Let $f \in \mathcal{R}(B(0 ; 1))$. Assume that for any non closed $C^{1}$ curve $\gamma$ with $\vec{\gamma} \neq \overrightarrow{0}$ for $t \in(0,1)$, one has that

$$
\gamma_{0}^{\prime}(t) \partial_{x_{0}} f_{0}(\gamma(t))+\left\langle\vec{\gamma}^{\prime}(t), \nabla f_{0}(\gamma(t))\right\rangle_{\mathbb{R}^{3}}>0, \quad \forall t \in(0,1)
$$


where $f=f_{0}+\vec{f}$ and $\nabla$ is the gradient operator in $\mathbb{R}^{3}$. Then $f$ is an univalent function in $B(0 ; 1)$.

Proof. Let $q_{1}, q_{2} \in B(0 ; 1)$ and let $\gamma$ be a $C^{1}$ curve in $B(0 ; 1)$ such that $\vec{\gamma}(t) \neq 0$ for any $t \in(0,1)$ and $\gamma(1)=q_{2}, \gamma(0)=q_{1}$. The fundamental theorem of calculus in one real variable implies

$$
f_{k}\left(q_{2}\right)-f_{k}\left(q_{1}\right)=\int_{0}^{1} \sum_{m=0}^{3} \gamma_{m}^{\prime}(t) \partial_{m}\left(f_{k}\right)(\gamma(t)) d t
$$

for $k=0,1,2,3$, where $\partial_{m}$ denotes $\partial_{x_{m}}$. Thus

$$
f\left(q_{2}\right)-f\left(q_{1}\right)=\int_{0}^{1} \sum_{m=0}^{3} \sum_{k=0}^{3} \gamma_{m}^{\prime}(t) \partial_{m}\left(f_{k}\right)(\gamma(t)) e_{k} d t
$$

and using the linearity of the partial derivatives one has

$$
f\left(q_{2}\right)-f\left(q_{1}\right)=\int_{0}^{1} \sum_{m=0}^{3} \gamma_{m}^{\prime}(t) \partial_{m}(f)(\gamma(t)) d t .
$$

As $f \in \mathcal{R}(B(0 ; 1))$, then $G[f]=0$ on $B(0 ; 1)$, where

$$
G[f](q)=\|\vec{q}\|^{2} \partial_{0} f(q)+\vec{q} \sum_{k=1}^{3} q_{m} \partial_{m} f(q)=0, \quad \forall q \in B(0 ; 1),
$$

see [1]. In particular,

$$
\partial_{0} f(\gamma(t))=-\frac{\vec{\gamma}(t)}{\|\vec{\gamma}(t)\|^{2}} \sum_{k=1}^{3} \gamma_{m}(t) \partial_{m} f(\gamma(t))=(\vec{\gamma}(t))^{-1} \sum_{k=1}^{3} \gamma_{m}(t) \partial_{m} f(\gamma(t)),
$$

for each $t \in(0,1)$. As

$$
\sum_{m=0}^{3} \gamma_{m}^{\prime}(t) \partial_{m}(f)(\gamma(t))=\gamma_{0}^{\prime}(t) \partial_{0}(f)(\gamma(t))+\sum_{m=1}^{3} \gamma_{m}^{\prime}(t) \partial_{m}(f)(\gamma(t)),
$$

and replacing $\partial_{0}(f)(\gamma(t))$ according to (3.2), one obtains

$$
\begin{gathered}
\sum_{m=0}^{3} \gamma_{m}^{\prime}(t) \partial_{m}(f)(\gamma(t))=\gamma_{0}^{\prime}(t)(\vec{\gamma}(t))^{-1} \sum_{m=1}^{3} \gamma_{m}(t) \partial_{m} f(\gamma(t))+\sum_{m=1}^{3} \gamma_{m}^{\prime}(t) \partial_{m}(f)(\gamma(t)) \\
=\sum_{m=1}^{3}\left[\gamma_{0}^{\prime}(t)(\vec{\gamma}(t))^{-1} \gamma_{m}(t)+\gamma_{m}^{\prime}(t)\right] \partial_{m}(f)(\gamma(t)), \quad \forall t \in(0,1) .
\end{gathered}
$$

The real part of (3.1) equals

$$
\begin{gathered}
\operatorname{Re}\left(f\left(q_{2}\right)-f\left(q_{1}\right)\right)=\operatorname{Re}\left(\int_{0}^{1} \sum_{m=0}^{3} \gamma_{m}^{\prime}(t) \partial_{m}(f)(\gamma(t)) d t\right) \\
\operatorname{Re}\left(f\left(q_{2}\right)-f\left(q_{1}\right)\right)=\operatorname{Re}\left(\int_{0}^{1} \sum_{k=1}^{3}\left[\gamma_{0}^{\prime}(t)(\vec{\gamma}(t))^{-1} \gamma_{m}(t)+\gamma_{m}^{\prime}(t)\right] \partial_{m}(f)(\gamma(t)) d t\right)
\end{gathered}
$$


$=\int_{0}^{1}\left(-\gamma_{0}^{\prime}(t)\left\langle\vec{\gamma}(t)^{-1}, \sum_{k=1}^{3} \gamma_{m}(t) \partial_{m}(\vec{f})(\gamma(t))\right\rangle+\sum_{k=1}^{3} \gamma_{m}^{\prime}(t) \partial_{m} f_{0}(\gamma(t))\right) d t$

Multiplying both sides of (3.2) by $\vec{\gamma}(t)$ and considering its vectorial part, we have

$$
\partial_{0} f_{0}(\gamma(t)) \vec{\gamma}(t)+\left[\vec{\gamma}(t) ; \partial_{0} \vec{f}(\gamma(t))\right]=\sum_{k=1}^{3} \gamma_{m}(t) \partial_{m}(\vec{f})(\gamma(t))
$$

Therefore

$\operatorname{Re}\left(f\left(q_{2}\right)-f\left(q_{1}\right)\right)=\int_{0}^{1}\left(-\gamma_{0}^{\prime}(t)\left\langle\vec{\gamma}(t)^{-1}, \partial_{0} f_{0}(\gamma(t)) \vec{\gamma}(t)\right\rangle+\sum_{k=1}^{3} \gamma_{m}^{\prime}(t) \partial_{m} f_{0}(\gamma(t))\right) d t$

or, equivalently,

$$
\begin{aligned}
& \operatorname{Re}\left(f\left(q_{2}\right)-f\left(q_{1}\right)\right)=\int_{0}^{1}\left(\gamma_{0}^{\prime}(t) \partial_{0} f_{0}(\gamma(t))+\sum_{k=1}^{3} \gamma_{m}^{\prime}(t) \partial_{m} f_{0}(\gamma(t))\right) d t \\
& \operatorname{Re}\left(f\left(q_{2}\right)-f\left(q_{1}\right)\right)=\int_{0}^{1}\left(\gamma_{0}^{\prime}(t) \partial_{0} f_{0}(\gamma(t))+\left\langle\vec{\gamma}^{\prime}(t), \vec{\Delta} f_{0}(\gamma(t))\right\rangle\right) d t
\end{aligned}
$$

Then $\operatorname{Re}\left(f\left(q_{2}\right)-f\left(q_{1}\right)\right)>0$ and thus $f$ is univalent.

Theorem 3.10. Let $f \in \mathcal{S} \cap \mathcal{N}(B(0 ; 1))$. We have:

(1) $B\left(0 ; \frac{1}{4}\right) \subset f(B(0 ; 1))$;

$$
\frac{1-r}{(1+r)^{3}} \leq\left\|\partial_{s} f(q)\right\| \leq \frac{1+r}{(1-r)^{3}}, \quad 0<\|q\|=r<1
$$

$$
\frac{r}{(1+r)^{2}} \leq\|f(q)\| \leq \frac{r}{(1-r)^{2}}, \quad 0<\|q\|=r<1
$$

$$
\frac{1-r}{(1+r)} \leq\left\|q \partial_{s} f(q) * f(q)^{-*}\right\| \leq \frac{1+r}{(1-r)}, \quad 0<\|q\|=r<1
$$

(5) For each $I \in \mathbb{S}$,

$$
r \int_{0}^{2 \pi}\left\|\partial_{s} f\left(r e^{I \theta}\right)\right\| d \theta \leq \frac{2 \pi r(1+r)}{(1-r)^{2}} .
$$

Proof. As $f \in \mathcal{N}(B(0 ; 1))$, since $f\left[B(0 ; 1) \cap \mathbb{C}_{I}\right] \subset \mathbb{C}_{I}$ for every $I \in \mathcal{S}$, we can use on each slice the usual complex theory. In fact, most of them are metrical properties which do not depend on the choice of $I$.

1) Apply Theorem 2.3 (Koebe One-Quarter Theorem) in e.g. [16. Note that since the quaternionic Koebe functions $K_{\theta}(q)=q \cdot\left[\left(1+q e^{I_{q} \theta}\right)^{2}\right]^{-1}, \theta \in \mathbb{R}$ are not, in general, intrinsic functions (except the cases $\theta=p \pi, p \in \mathbb{Z}$ ), we have only $B(0 ; 1 / 4) \subseteq \bigcap_{f \in \mathcal{S} \cap \mathcal{N}(B(0 ; 1))} f(B(0 ; 1))$ and not equality, as in the complex case. 
2) Apply Theorem 2.5 (Distortion Theorem) in e.g. [16] on each slice. As at the above point 2), the only intrinsic quternionic Koebe functions are $K_{0}(q)=q \cdot\left[(1+q)^{2}\right]^{-1}$ and $K_{\pi}(q)=q \cdot\left[(1-q)^{2}\right]^{-1}$, which attaint equalities in the obtained inequalities only at the points $q=r$ and $q=-r$. For this reason, we do not have the situation in the complex case, when the equalities are attained if and only if $f$ is a suitable chosen complex Koebe function.

3) Apply Theorem 2.6 (Growth Theorem) in e.g. [16] on each slice and the remark on the Koebe's functions from the point 2.

4) Apply Theorem 2.7 in e.g. [16] on each slice and again the remark on the Koebe's functions from the point 2.

5) Apply Theorem 2.9 in e.g. [16] on each slice.

According to Definition 2.2 in [9], we define another subset of slice regular functions:

$$
\mathcal{V}_{I}(B(0 ; 1))=\left\{f \in \mathcal{R}(B(0 ; 1)) ; f\left(B(0 ; 1) \cap \mathbb{C}_{I}\right) \subset \mathbb{C}_{I}\right\} .
$$

Therefore, we introduce the set

$$
\mathcal{V}(B(0 ; 1))=\bigcup_{I \in \mathcal{S}} \mathcal{V}_{I}(B(0 ; 1))
$$

It is clear that as $\mathcal{N}(B(0 ; 1))=\bigcap_{I \in \mathcal{S}} \mathcal{V}_{I}(B(0 ; 1))$, the set $\mathcal{V}(B(0 ; 1))$ is much larger than $\mathcal{N}(B(0 ; 1))$. According to Corollary 2.8 in 9 , we have $f \in$ $\mathcal{V}_{I}(B(0 ; 1))$ if and only if for all $q \in B(0 ; 1), f(q)=\sum_{k=0}^{\infty} q^{k} a_{k}$, with $a_{k} \in \mathbb{C}_{I}$ for all $k=0,1, \ldots$, .

We have the following result:

Theorem 3.11. Let $f \in \mathcal{S} \cap \mathcal{V}(B(0 ; 1))$. We have :

(1) If $f \in \mathcal{V}_{I}(B(0 ; 1))$ and $f(B(0 ; 1)) \subset \mathbb{H} \backslash \overline{B(0 ; 1)}$ is of the form

$$
f(q)=q+\sum_{n=0}^{\infty} q^{-n} a_{n}, \quad q \in B(0 ; 1),
$$

then the area of $f\left(B(0 ; 1) \cap \mathbb{C}_{I}\right)$ is

$$
2 \pi^{2}\left(1-\sum_{n=1} n\left|a_{n}\right|^{2}\right)
$$

(2) (Bieberbach-de Branges Theorem) If

$$
f(q)=q+\sum_{n=2}^{\infty} q^{n} a_{n}, \quad q \in B(0 ; 1),
$$

then $\left|a_{n}\right| \leq n$ for each $n=2,3,4, \ldots$.

Proof. 1) The function $f$, by assumption, has coefficients in $\mathbb{C}_{I}$. Theorem 2.1 (Area Theorem) in [16], gives that the area $A\left(f\left(\mathbb{B} \cap \mathbb{C}_{I}\right)\right)$ is

$$
A\left(f\left(\mathbb{B} \cap \mathbb{C}_{I}\right)\right)=\pi\left(1-\sum_{n=1} n\left|a_{n}\right|^{2}\right) .
$$


2) Since the coefficients of $f$ belongs to a given complex plane $\mathbb{C}_{I}$, according to the result in e.g. [13] applied on a chosen slice, we get the corresponding estimates for the coefficients of the series development of $f$.

Remark 3.12. It is worth noting that for any fixed $I \in \mathcal{S}$, the set $\mathcal{V}_{I}(B(0 ; 1))$ can be generated from the whole class of univalent analytic functions of complex variable in the unit disk, by simply replacing in each such complex function $z$ by $q$, and in the coefficients of the series development, the small $i$ with the capital $I$. In this way, any univalent analytic function of complex variable in the unit disk with at least one non-real coefficient in its series development, generates an infinity of quaternionic functions in $\mathcal{V}(B(0 ; 1))$.

Open Question. It is a natural question if $\mathcal{V}(B(0 ; 1))$ is the largest class of univalent slice regular functions in which the Bieberbach - de Branges result holds. There could exists $f \in \mathcal{S}$ which is not in $\mathcal{V}(B(0 ; 1))$, for which this result does not hold.

Algebraic and Geometric Interpretations. Some results of Theorem 3.10, can be interpreted as geometric or algebraic properties of infinite differentiable injective transformations from $\mathbb{R}^{4}$ to $\mathbb{R}^{4}$. Indeed, any $f: B(0,1) \rightarrow \mathbb{H}$ can be written in the form

$$
f\left(x_{1}+i x_{2}+j x_{3}+k x_{4}\right)
$$

$=P_{1}\left(x_{1}, x_{2}, x_{3}, x_{4}\right)+i P_{2}\left(x_{1}, x_{2}, x_{3}, x_{4}\right)+j P_{3}\left(x_{1}, x_{2}, x_{3}, x_{4}\right)+k P_{4}\left(x_{1}, x_{2}, x_{3}, x_{4}\right)$, with all $P_{k}\left(x_{1}, x_{2}, x_{3}, x_{4}\right), k=1,2,3,4$, real-valued, and therefore $f$ can be also be viewed as the transformation

$$
\begin{gathered}
\left(x_{1}, x_{2}, x_{3}, x_{4}\right) \rightarrow \\
\left(P_{1}\left(x_{1}, x_{2}, x_{3}, x_{4}\right), P_{2}\left(x_{1}, x_{2}, x_{3}, x_{4}\right), P_{3}\left(x_{1}, x_{2}, x_{3}, x_{4}\right), P_{4}\left(x_{1}, x_{2}, x_{3}, x_{4}\right)\right) .
\end{gathered}
$$

Below we present a few illustrations.

1) In the case of Theorem 3.10, 2), a first geometric interpretation is the relationship

$$
B(0 ; 1 / 4) \subseteq \bigcap_{f \in \mathcal{S} \cap \mathcal{N}(B(0 ; 1))} f[B(0 ; 1)]
$$

Note that due to the fact that most of the quaternionic Koebe functions are not intrinsic functions, the situation is different from the complex case, when above instead of $\subseteq$ we have equality.

Secondly, by identifying $q=x_{1}+i x_{2}+j x_{3}+k x_{4} \in \mathbb{H}$ with $\left(x_{1}, x_{2}, x_{3}, x_{4}\right)$ and defining the Euclidean distance in $\mathbb{R}^{4}$ by $d_{\mathbb{R}^{4}}^{(E)}(0, q)=\sqrt{x_{1}^{2}+x_{2}^{2}+x_{3}^{2}+x_{4}^{2}}$, the inclusion in Theorem [3.10, 2), can be written as follows : if $f \in$ $\mathcal{S} \cap \mathcal{N}(B(0 ; 1))$ then for any $r \in(0,1 / 4)$, there exists $q$ with $d_{\mathbb{R}^{4}}^{(E)}(0, q)<1$ solution of the equation $d_{\mathbb{R}^{4}}^{(E)}(0, f(q))=r$. For example, if we consider $f(q)=q+\frac{q^{2}}{4}$ which is in $\mathcal{S} \cap \mathcal{N}(B(0 ; 1))$, since by simple calculation we get $f=P_{1}+i P_{2}+j P_{3}+k P_{4}$ where $P_{1}=x_{1}+\frac{1}{4}\left(x_{1}^{2}-x_{2}^{2}-x_{3}^{2}-x_{4}^{2}\right)$, $P_{2}=x_{2}+\frac{x_{1} x_{2}}{2}, P_{3}=x_{3}+\frac{x_{1} x_{3}}{2}, P_{4}=x_{4}+\frac{x_{1} x_{4}}{2}$, it follows that for any $r \in(0,1 / 4)$, the algebraic equation $P_{1}^{2}+P_{2}^{2}+P_{3}^{2}+P_{4}^{2}=r^{2}$ has at least 
one solution $\left(x_{1}, x_{2}, x_{3}, x_{4}\right)$ with $x_{1}^{2}+x_{2}^{2}+x_{3}^{3}+x_{4}^{2}<1$. It is clear that the attempt to solve this algebraic equation by direct methods is not an easy task.

2) In the case of Theorem 3.10, 3), denoting by $R_{1}=\frac{r}{(1+r)^{2}}$ and $R_{2}=\frac{r}{(1-r)^{2}}$, a geometric interpretation of the inequalities is as follows :

$$
B\left(0 ; R_{1}\right) \subseteq \bigcap_{f \in \mathcal{S} \cap \mathcal{N}(B(0 ; 1))} f[B(0 ; r)], \quad \bigcup_{f \in \mathcal{S} \cap \mathcal{N}(B(0 ; 1))} f[B(0 ; r)] \subseteq B\left(0 ; R_{2}\right) .
$$

Note again that due to the fact that most of the quaternionic Koebe functions are not intrinsic functions, the situation is different from the complex case, when above instead of $\subseteq$ we have equalities.

Also, with the notations from the above point, the inequalities in Theorem 3.10, 3), can be written as follows : for any $q$ with $0<d_{\mathbb{R}^{4}}^{(E)}(0, q)<1$ we have

$$
\frac{1-d_{\mathbb{R}^{4}}^{(E)}(0, q)}{\left(1+d_{\mathbb{R}^{4}}^{(E)}(0, q)\right)^{3}} \leq d_{\mathbb{R}^{4}}^{(E)}\left(0, \partial_{s} f(q)\right) \leq \frac{1+d_{\mathbb{R}^{4}}^{(E)}(0, q)}{\left(1-d_{\mathbb{R}^{4}}^{(E)}(0, q)\right)^{3}} .
$$

Similarly, the inequalities in Theorem 3.10 , 4) can be written as

$$
\frac{d_{\mathbb{R}^{4}}^{(E)}(0, q)}{\left(1+d_{\mathbb{R}^{4}}^{(E)}(0, q)\right)^{2}} \leq d_{\mathbb{R}^{4}}^{(E)}(0, f(q)) \leq \frac{d_{\mathbb{R}^{4}}^{(E)}(0, q)}{\left(1-d_{\mathbb{R}^{4}}^{(E)}(0, q)\right)^{2}},
$$

for any $q$ with $0<d_{\mathbb{R}^{4}}^{(E)}(0, q)<1$.

For particular functions $f$ as, for example, $f(q)=q+\frac{q^{2}}{4}$, these relationships become algebraic inequalities which are not easy to be proved by direct methods.

Theorem 3.13. (Area Theorem) Let $f$ be a slice regular function on $\Delta:=$ $\{q \in \mathbb{H} \mid\|q\|>1\}$, such that

$$
f(q)=q+\sum_{n=0}^{\infty} q^{-n} a_{n}
$$

Assume that for some $I \in \mathbb{S}$ and $J \in \mathbb{S}$ orthogonal to $I$, one has $f_{\mathbb{D}_{I}}=$ $f_{1}+f_{2} J$ where $f_{1}(z)$ and $z+f_{2}(z)$ are univalent functions. Then

$$
\left(\left[\frac{1}{2}(f-I f I)\left(\Delta_{I}\right)\right]^{c}\right)+m\left(\left[\left(\mathcal{I}+\frac{1}{2}(f+I f I) \bar{J}\right)\left(\Delta_{I}\right)\right]^{c}\right)=\pi\left(2-\sum_{n=1}^{\infty} n\left\|a_{n}\right\|^{2}\right) .
$$

where $\Delta_{I}=\Delta \cap \mathbb{C}_{I}, \mathcal{I}$ is the identity function on $B(0 ; 1)$ and $m$ is the two dimensional outer measure. Here $A^{c}$ denotes $\mathbb{H} \backslash A$.

(2) We have the inequality

$$
\sum_{n=1}^{\infty} n\left\|a_{n}\right\|^{2} \leq 2
$$

(3) We have $\left\|a_{1}\right\| \leq \sqrt{2}$, with equality if and only if $f(q)=q+a_{0}+a_{1} q^{-1}$. 
Proof. (1) Let $I, J \in \mathbb{S}$ with $J \perp I$ and let $f_{1}, f_{2}$ be holomorphic functions on $\Delta_{I}$ such that $\left.f\right|_{\Delta_{I}}=f_{1}+f_{2} J$ such that $f_{1}(z)$ and $z+f_{2}(z)$ are univalent functions. Moreover, if $a_{n}=a_{1, n}+a_{2, n} J$ with $a_{k, n} \in \mathbb{C}_{I}$ for each $k=1,2$ and any $n \in \mathbb{N} \cup\{0\}$, then

$$
f_{1}(z)=z+\sum_{n=0}^{\infty} z^{-n} a_{1, n}, \quad z+f_{2}(z)=z+\sum_{n=0}^{\infty} z^{-n} a_{2, n},
$$

on $\Delta_{I}$. Thus, since the above functions $f_{1}, \mathcal{I}+f_{2}$ have a simple pole at infinity with residue 1 (where $\mathcal{I}$ represents the identity function on $B(0 ; 1)$ ), from Theorem 1.2, p. 29 in [16] and observing that $f_{1}=\left.\frac{1}{2}(f-I f I)\right|_{\Delta_{I}}$, $f_{2}=\left.\frac{1}{2}(f+I f I) \bar{J}\right|_{\Delta_{I}}, \quad a_{1, n}=\frac{1}{2}\left(a_{n}-I a_{n} I\right)$ and $a_{2, n}=\frac{1}{2}\left(a_{n}+I a_{n} I\right) \bar{J}$, for each $n \in \mathbb{N} \cup\{0\}$, one has that

$$
\begin{gathered}
m\left(\left[\frac{1}{2}(f-I f I)\left(\Delta_{I}\right)\right]^{c}\right)=\pi\left(1-\frac{1}{4} \sum_{n=1}^{\infty} n\left\|a_{n}-I a_{n} I\right\|^{2}\right), \\
m\left(\left[\left(\mathcal{I}+\frac{1}{2}(f+I f I) \bar{J}\right)\left(\Delta_{I}\right)\right]^{c}\right)=\pi\left(1-\frac{1}{4} \sum_{n=1}^{\infty} n\left\|a_{n}+I a_{n} I\right\|^{2}\right) .
\end{gathered}
$$

Finally, from the previous formula and the parallelogram identity:

$$
\left\|a_{n}-I a_{n} I\right\|^{2}+\left\|a_{n}+I a_{n} I\right\|^{2}=2\left\|a_{n}\right\|^{2}+2\left\|I a_{n} I\right\|^{2}=4\left\|a_{n}\right\|^{2},
$$

for each $n \in \mathbb{N}$, one concludes

$$
m\left(\left[\frac{1}{2}(f-I f I)\left(\Delta_{I}\right)\right]^{c}\right)+m\left(\left[\left(\mathcal{I}+\frac{1}{2}(f+I f I) \bar{J}\right)\left(\Delta_{I}\right)\right]^{c}\right)=\pi\left(2-\sum_{n=1}^{\infty} n\left\|a_{n}\right\|^{2}\right) .
$$

Point (2) follows from (1) and (3) is an immediate consequence of (2).

A variation of the previous result is the following:

Theorem 3.14. Let $f \in \mathcal{R}(B(0 ; 1)), f(q)=\sum_{n=0}^{\infty} q^{n} a_{n}$ and let $f_{1}, f_{2} \in$ $\operatorname{Hol}\left(\mathbb{D}_{I}\right)$ be such that $\left.f\right|_{D_{I}}=f_{1}+f_{2} J$. Assume that the functions $z+$ $f_{1}\left(z^{-1}\right), \quad z+f_{2}\left(z^{-1}\right)$ are univalent functions on $\Delta_{I}:=\left\{z \in \mathbb{C}_{I}|| z \mid>1\right\}$. Then

$$
\sum_{n=1}^{\infty} n\left\|a_{n}\right\|^{2} \leq 2
$$

Proof. From our assumptions it follows that

$$
\left.f\right|_{\mathbb{D}_{I}}\left(z^{-1}\right)=\sum_{n=0}^{\infty} z^{-n} a_{n}, \quad z \in \Delta_{I}
$$

and if $a_{n}=a_{1, n}+a_{2, n} J$ with $a_{1, n}, a_{2, n} \in \mathbb{C}_{I}$ for each $n \in \mathbb{N} \cup\{0\}$, then

$$
z+f_{1}\left(z^{-1}\right)=z+\sum_{n=0}^{\infty} z^{-n} a_{1, n}, \quad z+f_{2}\left(z^{-1}\right)=z+\sum_{n=0}^{\infty} z^{-n} a_{2, n} .
$$


Thus repeating the computations in proof of Theorem 3.13 one obtains that

$$
\sum_{n=1}^{\infty} n\left\|a_{n}\right\|^{2} \leq 2 .
$$

Remark 3.15. As a direct consequence of previous result one has that

$$
\left\|a_{1}\right\| \leq \sqrt{2}, \quad\left\|a_{2}\right\| \leq 1 .
$$

Note that if $a_{1}=1$ then

$$
\sum_{n=2}^{\infty} n\left\|a_{n}\right\|^{2} \leq 1 .
$$

For $t \in(-1,1)$ denote

$$
T_{t}(q)=\frac{q+t}{1+t q}, \quad q \in B(0 ; 1) .
$$

We have the following result:

Proposition 3.16. Let $f \in \mathcal{R}(B(0 ; 1))$. Let $I, J \in \mathbb{S}$, $J$ orthogonal to $I$, and $\left.f\right|_{\mathbb{D}_{I}}=f_{1}+f_{2} J$ with $f_{1}, f_{2} \in \operatorname{Hol}\left(\mathbb{D}_{I}\right)$. If there exists $0<\delta<1$ such that

$$
z+f_{1} \circ T_{t}\left(z^{-1}\right), \quad z+f_{2} \circ T_{t}\left(z^{-1}\right), \quad z \in \Delta_{I}
$$

are univalent functions on $\Delta_{I}$ for each $t \in(-\delta, \delta)$, then

$$
\left\|\partial_{s} f(t)\right\| \leq \frac{\sqrt{2}}{1-t^{2}}
$$

and

$$
\left\|-2 t \partial_{s} f(t)+\left(1-t^{2}\right) \partial_{s}^{2} f(t)\right\| \leq \frac{1}{1-t^{2}}
$$

for each $t \in(-\delta, \delta)$.

Proof. As $g=f \circ T_{t} \in \mathcal{R}(B(0 ; 1))$ and it satisfies the conditions of previous proposition, then denoting

$$
g \circ T_{t}^{-1}(q)=\sum_{n=0}^{\infty} q^{n} a_{n},
$$

one has that $\left\|a_{1}\right\|^{2} \leq 2$ and $\left\|a_{2}\right\|^{2} \leq 1$. Note also that

$$
a_{1}=\partial_{s} g(0)=\partial_{s} T_{t}(0) \partial_{s} f\left(T_{t}(0)\right)=\left(1-t^{2}\right) \partial_{s} f(t)
$$

and

$$
\begin{gathered}
a_{2}=\frac{1}{2} \partial_{s}^{2} g(0)=\partial_{s}^{2} T_{t}(0) \partial_{s} f\left(T_{t}(0)\right)+\left(\partial_{s} T_{t}(0)\right)^{2} \partial_{s}^{2} f\left(T_{t}(0)\right)= \\
=-2\left(1-t^{2}\right) t \partial_{s} f(t)+\left(1-t^{2}\right)^{2} \partial_{s}^{2} f(t)
\end{gathered}
$$

Therefore

$$
\left\|\left(1-t^{2}\right) \partial_{s} f(t)\right\|^{2} \leq 2
$$


and

$$
\left\|-2\left(1-t^{2}\right) t \partial_{s} f(t)+\left(1-t^{2}\right)^{2} \partial_{s}^{2} f(t)\right\|^{2} \leq 1
$$

for each $t \in(-\delta, \delta)$.

\section{Subordination of Slice Regular Functions}

In this section firstly we introduce and study a notion of composition of slice regular functions which is of independent interest, but which also will be used to study the subordination concept between two slice regular functions. As it is well know, see for example [8], the composition $f \circ g$ of two slice regular functions is not anymore slice regular, unless $g$ belongs to the subclass $\mathcal{N}$, i.e., it is quaternionic intrinsic. However, in order to define the notion of subordination it is necessary to have a notion of composition between slice regular functions. To motivate our choice of the notion of composition, we also recall that the pointwise product does not preserve slice regularity (unless one of the functions belongs to $\mathcal{N}$ ) and while the slice regularity is preserved by the so-called $*$-product. The power of a function is slice regular only if it is computed with respect to the slice product and we will write $(w(q))^{* n}$ when we take the $n$-th power with respect to the *product. This justifies the definition below. We first treat the case of formal power series.

Definition 4.1. Denoting $g(q)=\sum_{n=0}^{\infty} q^{n} a_{n}$ and $w(q)=\sum_{n=1}^{\infty} q^{n} b_{n}$. We define

$$
(g \bullet w)(q)=\sum_{n=0}^{\infty}(w(q))^{* n} a_{n} .
$$

Remark 4.2. Note that if $w \in \mathcal{N}(B(0 ; 1))$, then $g \bullet w$ becomes $g \circ w$ where $\circ$ represents the usual composition of two functions. Note that if $w$ is quaternionic intrinsic $(w(q))^{* n}=(w(q))^{n}$ so, in particular, $q^{* n}=q^{n}$.

Remark 4.3. Following [7] we call order of a series $f(q)=\sum_{n=0}^{\infty} q^{n} a_{n}$ and we denote it by $\omega(f)$, the least integer $n$ such that $a_{n} \neq 0$ (with the convention that the order of the series identically equal to zero is $+\infty$ ). Assume to have a family $\left\{f_{i}\right\}_{i \in \mathcal{I}}$ of power series where $\mathcal{I}$ is a set of indices. The family is said to be summable if for any $k \in \mathbb{N}, \omega\left(f_{i}\right) \geq k$ for all except a finite number of indices $i$. By definition the sum of $\left\{f_{i}\right\}$ where $f_{i}(q)=\sum_{n=0}^{\infty} q^{n} a_{i, n}$ is

$$
f(q)=\sum_{n=0}^{\infty} q^{n} a_{n},
$$

where $a_{n}=\sum_{i \in \mathcal{I}} a_{i, n}$. Note that the definition of $a_{n}$ makes sense since our hypothesis guarantees that for any $n$ just a finite number of $a_{i, n}$ are nonzero.

Remark 4.4. The hypothesis $b_{0}=0$ in Definition 4.1 is necessary in order to guarantee that in the term $(w(q))^{* n}$ the minimum power of $q$ is at least $q^{n}$ or, in other words, that $\omega\left(w(q)^{* n}\right) \geq n$ (for all indices). In this way, the 
series $\sum_{n=0}^{\infty}(w(q))^{* n} a_{n}$ is summable, see Remark 4.3, and we can regroup the powers of $q$.

This composition, in general it is not associative as one can directly verify with an example: by taking $f(q)=q^{2} c, g(q)=q a$ and $w(q)=q^{2} b$ we have $((f \bullet g) \bullet w)=q^{4} b^{2} a^{2} c$ while $(f \bullet(g \bullet w))(q)=q^{4} b a b a c$. However, we will prove that the composition is associative in some cases and to this end we need a preliminary Lemma.

Lemma 4.5. Let $f_{1}(q)=\sum_{n=0}^{\infty} q^{n} a_{n}, f_{2}(q)=\sum_{n=0}^{\infty} q^{n} b_{n}$, and $g(q)=$ $\sum_{n=1}^{\infty} q^{n} c_{n}$. Then:

(1) $\left(f_{1}+f_{2}\right) \bullet g=f_{1} \bullet g+f_{2} \bullet g$;

(2) if $g$ has real coefficients $\left(f_{1} * f_{2}\right) \bullet g=\left(f_{1} \bullet g\right) *\left(f_{2} \bullet g\right)$;

(3) if $g$ has real coefficients $f^{* n} \bullet g=(f \bullet g)^{* n}$.

Moreover, if $\left\{f_{i}\right\}_{i \in \mathcal{I}}$ is a summable family of power series then $\left\{f_{i} \bullet w\right\}_{i \in \mathcal{I}}$ is summable and

(1) $\left(\sum_{i} f_{i}\right) \bullet w=\sum_{i}\left(f_{i} \bullet w\right)$.

Proof. To prove (1) observe that

$$
\left(f_{1}+f_{2}\right) \bullet g=\sum_{n=0}^{\infty} g^{* n}\left(a_{n}+b_{n}\right)=\sum_{n=0}^{\infty} g^{* n} a_{n}+\sum_{n=0}^{\infty} g^{* n} b_{n}=f_{1} \bullet g+f_{2} \bullet g .
$$

Let us prove (2). We have $f_{1} * f_{2}(q)=\sum_{n=0}^{\infty} q^{n}\left(\sum_{r=0}^{n} a_{r} b_{n-r}\right)$ and so

$$
\left(\left(f_{1} * f_{2}\right) \bullet g\right)(q)=\sum_{n=0}^{\infty}(g(q))^{n}\left(\sum_{r=0}^{n} a_{r} b_{n-r}\right)
$$

and, taking into account that the coefficients of $g$ are real:

$$
\left(f_{1} \bullet g\right)(q) *\left(f_{2} \bullet g\right)(q)=\left(\sum_{n=0}^{\infty}\left(g(q)^{n} a_{n}\right) *\left(\sum_{m=0}^{\infty} g(q)^{m} b_{m}\right)=\sum_{n=0}^{\infty} g(q)^{n}\left(\sum_{r=0}^{n} a_{r} b_{n-r}\right) .\right.
$$

We prove (3) by induction. Observe that the statement is true for $n=2$ since it follows from (2). Assume that the assertion is true for the $n$-th power. Let us show that it holds for $n+1$. Let us compute

$$
\left(f^{*(n+1)} \bullet g\right)(q)=\left(\left(f^{*(n)} * f\right) \bullet g\right)(q) \stackrel{(2)}{=}(f \bullet g)^{* n} *(f \bullet g)=(f \bullet g)^{*(n+1)},
$$

and the statement follows.

Finally, to show (4) we follow [7, p. 13]. Let $f_{i}(q)=\sum_{n=0}^{\infty} q^{n} a_{i, n}$ so that we have, by definition

$$
\sum_{i \in \mathcal{I}} f_{i}(q)=\sum_{n=0}^{\infty} q^{n}\left(\sum_{i \in \mathcal{I}} a_{i, n}\right)
$$

Thus we obtain

$$
\left(\sum_{i \in \mathcal{I}} f_{i}(q)\right) \bullet g=\sum_{n=0}^{\infty} g(q)^{* n}\left(\sum_{i \in \mathcal{I}} a_{i, n}\right)
$$


and

$$
\sum_{i \in \mathcal{I}}\left(f_{i} \bullet g\right)(q)=\sum_{i \in \mathcal{I}}\left(\sum_{n=0}^{\infty} g(q)^{* n} a_{i, n}\right)
$$

Now observe that, by hypothesis on the summability of $\left\{f_{i}\right\}$, each power of $q$ involves just a finite number of the coefficients $a_{i, n}$ so we can apply the associativity of the addition in $\mathbb{H}$ and so (4.1) and (4.2) are equal.

Proposition 4.6. If $f(q)=\sum_{n=0}^{\infty} q^{n} c_{n}, g(q)=\sum_{n=1}^{\infty} q^{n} a_{n}, w(q)=\sum_{n=1}^{\infty} q^{n} b_{n}$ and $w$ has real coefficients, then $(f \bullet g) \bullet w=f \bullet(g \bullet w)$.

Proof. We adapt the proof of Proposition 4.1 in [7]. Let us begin by proving the equality in the special case in which $f(q)=q^{n} a_{n}$. We have:

$$
((f \bullet g) \bullet w)(q)=\left(g^{* n} \bullet w\right) a_{n}
$$

and

$$
(f \bullet(g \bullet w))(q)=(g \bullet w)^{* n}(q) a_{n} .
$$

Lemma 4.5, (3) shows that $\left(g^{* n} \bullet w\right)=\left(g^{* n} \bullet w\right)$ and the equality follows. The general case follows by considering $f$ as the sum of the summable family $\left\{q^{n} a_{n}\right\}$ and using the first part of the proof:

$$
(f \bullet g) \bullet w=\sum_{n=0}^{\infty}\left(g^{* n} \bullet w\right) a_{n}=\sum_{n=0}^{\infty}(g \bullet w)^{* n} a_{n}=f \bullet(g \bullet w) .
$$

So far, we have considered power series without specifying their set of convergence. We now take care of this aspect, by proving the following result which is classical for power series with coefficients in a commutative ring, see [7].

Proposition 4.7. Let $g(q)=\sum_{n=0}^{\infty} q^{n} a_{n}$ and $f(q)=\sum_{n=1}^{\infty} q^{n} b_{n}$ be convergent in the balls of nonzero radius $R$ and $\rho$, respectively, and let $h(q)=$ $(g \bullet f)(q)$. Then the radius of convergence of $h$ is nonzero and if $r>0$ is any real number such that $\sum_{n=1}^{\infty} r^{n}\left\|b_{n}\right\|<R$, then the radius of convergence of $h$ is greater than or equal $r$.

Proof. First of all, let us observe that

$$
\left\|\left(\sum_{m=1}^{\infty} q^{m} b_{m}\right)^{* n}\right\| \leq\left(\sum_{m=1}^{\infty}\|q\|^{m}\left\|b_{m}\right\|\right)^{n} .
$$


In fact, we have

$$
\begin{aligned}
\left\|\left(\sum_{m=1}^{\infty} q^{m} b_{m}\right) *\left(\sum_{m=1}^{\infty} q^{m} c_{m}\right)\right\| & =\left\|\sum_{m_{1}}^{\infty} q^{m}\left(\sum_{r=0}^{m} b_{r} c_{m-r}\right)\right\| \\
& \leq \sum_{m_{1}}^{\infty}\|q\|^{m}\left(\sum_{r=0}^{m}\left\|b_{r}\right\|\left\|c_{m-r}\right\|\right. \\
& =\left(\sum_{m=1}^{\infty}\|q\|^{m}\left\|b_{m}\right\|\right)\left(\sum_{m=1}^{\infty}\|q\|^{m}\left\|c_{m}\right\|\right),
\end{aligned}
$$

and so the statement is true for $n=2$ the rest follows recursively by using (4.3). So we have

$$
\begin{aligned}
\left\|\sum_{n=0}^{\infty}\left(\sum_{m=1}^{\infty} q^{m} b_{m}\right)^{* n} a_{n}\right\| & \leq \sum_{n=0}^{\infty}\left\|\left(\sum_{m=1}^{\infty} q^{m} b_{m}\right)^{* n}\right\|\left\|a_{n}\right\| \\
& \leq \sum_{n=0}^{\infty}\left(\sum_{m=1}^{\infty}\|q\|^{m}\left\|b_{m}\right\|\right)^{n}\left\|a_{n}\right\|
\end{aligned}
$$

Now, since the series expressing $f$ is converging on a ball of finite radius, there exists a positive number $r$ such that $\sum_{n=1}^{\infty} r^{n}\left\|b_{n}\right\|$ is finite. Moreover, $\sum_{n=1}^{\infty} r^{n}\left\|b_{n}\right\|=r \sum_{n=1}^{\infty} r^{n-1}\left\|b_{n}\right\| \rightarrow 0$ for $r \rightarrow 0$ and so there exists $r$ such that $\sum_{n=1}^{\infty} r^{n}\left\|b_{n}\right\|<R$. Thus, from (4.4), we have that

$$
\sum_{n=0}^{\infty}\left(\sum_{m=1}^{\infty} r^{m}\left\|b_{m}\right\|\right)^{n}\left\|a_{n}\right\|=\sum_{n=0}^{\infty} \sum_{m=1}^{\infty} r^{m} \gamma_{m}<\infty .
$$

Thus we have that $(g \bullet f)(q)=\sum_{m=0}^{\infty} q^{m} c_{m}$ and $\left\|c_{m}\right\| \leq \gamma_{m}$ and thus the radius of convergence of $g \bullet f$ is at least equal to $r$, where $r$ is a sufficiently small positive real number.

We are now ready to give the notion of subordination:

Definition 4.8. Let $f, g$ be (left) slice regular on $B(0 ; 1)$.

(1) We say that $f$ is subordinated to $g$ and we write $f \prec g$ if there exists $w \in \mathcal{R}(B(0 ; 1))$ with $w(0)=0$ and $\|w(q)\|<1$, for all $q \in B(0 ; 1)$ such that $f(q)=(g \bullet w)(q)$ for all $q \in B(0 ; r)$, where $r$ is a suitable number in $(0,1)$.

(2) We write $f \prec_{\mathcal{N}} g$ if there exists $w \in \mathcal{N}(B(0 ; 1))$ with $w(0)=0$ and $\|w(q)\|<1$, for all $q \in B(0 ; 1)$ such that $f(q)=(g \bullet w)(q)$ for all $q \in B(0 ; 1)$.

(3) Let $I \in \mathbb{S}$, then $f$ is $I$-subordinated to $g$ and we write $f \prec_{I} g$ if there exists $w_{I} \in \operatorname{Hol}\left(\mathbb{D}_{I}\right)$ such that $\left|w_{I}(z)\right| \leq|z|$ for all $z \in \mathbb{D}_{I}$ and $\left.f\right|_{\mathbb{D}_{I}}(z)=\left(\left.g\right|_{\mathbb{D}_{I}} \circ w_{I}\right)(z)$ for all $z \in \mathbb{D}_{I}$.

Note that if $f \prec_{\mathcal{N}} g$ then $f \prec_{I} g$ for each $I \in \mathbb{S}$, but the converse is false, for example: $f(q)=q I, \quad g(q)=q$ for each $q \in B(0 ; 1)$ and $w_{I}(z)=z I$ for $z \in \mathbb{D}_{I}$ 
Theorem 4.9. Let $f, g \in \mathcal{R}(B(0 ; 1))$ and let $I \in \mathbb{S}$ such that $f \prec_{I} g$.

(1) Then $\left\|\partial_{s} f(0)\right\| \leq\left\|\partial_{s} g(0)\right\|$.

(2) If $g_{1}, g_{2}, g_{3}, g_{4} \in \mathcal{N}(B(0 ; 1))$ are such that $g=g_{1}+g_{2} I+g_{3} J+g_{4} I J$, with $J \in \mathbb{S}$ and $J \perp I$, then for any $q \in B(0 ; 1)$ there exist $q_{1}, q_{2} \in$ $B(0 ; 1)$ satisfying

$$
f(q)=g_{1}\left(q_{1}\right)+g_{2}\left(q_{1}\right) I+g_{3}\left(q_{2}\right) J+g_{4}\left(q_{2}\right) I J .
$$

Proof.

(1) Set $\left.f\right|_{\mathbb{D}_{I}}=v_{1}+v_{2} J$ and $\left.g\right|_{\mathbb{D}_{I}}=h_{1}+h_{2} J$ where $v_{1}, v,_{2}, h_{1}, h_{2} \in$ $\operatorname{Hol}\left(\mathbb{D}_{I}\right)$ and $J \in \mathbb{S}, J \perp I$. From the complex case, see [16], one has

$$
\begin{gathered}
\left\|\partial_{s} f(0)\right\|^{2}=\left\|\left.\partial_{s} f\right|_{\mathbb{D}_{I}}(0)\right\|^{2}=\left\|\partial_{s} v_{1}(0)\right\|^{2}+\left\|\partial_{s} v_{2}(0)\right\|^{2} \\
\leq\left\|\partial_{s} h_{1}(0)\right\|^{2}+\left\|\partial_{s} h_{2}(0)\right\|^{2}=\left\|\partial_{s} g(0)\right\|^{2} .
\end{gathered}
$$

(2) From previous notations we have that $h_{1}=g_{1}\left|\mathbb{D}_{I}+g_{2}\right|_{\mathbb{D}_{I}} I$ and $h_{2}=$ $g_{3}\left|\mathbb{D}_{I}+g_{4}\right|_{\mathbb{D}_{I}} I$ and as $f \prec_{I} g$ implies, in the theory of functions of one complex variable, $v_{1} \prec h_{1}$ and $v_{2} \prec h_{2}$. Subordination principle, see [16], implies each $z \in \mathbb{D}_{I}$ that there exist $z_{1}, z_{2} \in \mathbb{D}_{I}$ such that $f(z)=g_{1}\left(z_{1}\right)+g_{2}\left(z_{1}\right) I+g_{3}\left(z_{2}\right) J+g_{4}\left(z_{2}\right) I J$. The main result is obtained using the representation theorem for slice regular functions.

Definition 4.10. Given $f \in \mathcal{R}(B(0 ; 1))$ and let $I \in \mathbb{S}, 0 \leq r<1$ and $1 \leq p<\infty$. Set

(1) $M_{\infty}(r, f):=\max \{\|f(q)\| \mid q \in \mathbb{H},\|q\|=r\}$.

(2) $M_{p}(r, f):=\left[\frac{1}{4 \pi} \int_{\partial B(0 ; r)}\|f(q)\|^{p} d \mu\right]^{\frac{1}{p}}$, where $\partial B(0 ; r)=\{q \in \mathbb{H}$ $\|q\|=r\}$.

(3) $M_{\infty, I}(r, f):=\max \left\{\|f(q)\| \mid q \in \mathbb{D}_{I},\|q\|=r\right\}$.

(4) $M_{p, I}(r, f):=\left[\frac{1}{2 \pi} \int_{0}^{2 \pi}\left\|f\left(r e^{I \theta}\right)\right\|^{p} d \theta\right]^{\frac{1}{p}}$.

Theorem 4.11. Let $f, g \in \mathcal{R}(B(0 ; 1))$ with $f(0)=g(0)$, such that there exists $I \in \mathbb{S}$ with $f \prec_{I} g$. Then

(1) $M_{\infty, I}(r, f) \leq \sqrt{2} M_{\infty, I}(r, g)$.

(2) $M_{p, I}(r, f) \leq 2^{p+1} M_{p, I}(r, g)$.

(3) $M_{\infty}(r, f) \leq \sqrt{2} M_{\infty, I}(r, g) \leq \sqrt{2} M_{\infty}(r, g)$.

(4) $M_{p}(r, f) \leq 2^{2 p+2} \pi^{2} M_{p, I}(r, g)$.

Proof. All the properties are consequences of Littlewood's Subordination Theorem and the domination of the maximum modulus, see [16], and also of some inequalities that we prove below:

a) $\|f(z)\|^{2}=\left\|f_{1}(z)\right\|^{2}+\left\|f_{2}(z)\right\|^{2} \leq M_{\infty, I}\left(r, f_{1}\right)^{2}+M_{\infty, I}\left(r, f_{2}\right)^{2}$

$$
\leq M_{\infty, I}\left(r, g_{1}\right)^{2}+M_{\infty, I}\left(r, g_{2}\right)^{2} \leq 2 M_{\infty, I}(r, g)^{2}, \quad \forall z \in \mathbb{D}_{I},
$$


ON SOME GEOMETRIC PROPERTIES OF SLICE REGULAR FUNCTIONS

where $\left.f\right|_{\mathbb{D}_{I}}=f_{1}+f_{2} J$ and $\left.g\right|_{\mathbb{D}_{I}}=g_{1}+g_{2} J$ with $f_{1}, f_{2}, g_{1}, g_{2} \in \operatorname{Hol}\left(\mathbb{D}_{I}\right)$ and as $f \prec_{I} g$ then $f_{k} \prec_{I} g_{k}$ for $k=1,2$.

b) $\|f(z)\|^{p} \leq 2^{p}\left(\left\|f_{1}(z)\right\|^{p}+\left\|f_{2}(z)\right\|^{p}\right), \quad \forall z \in \mathbb{D}_{I}$.

c) In the following inequalities the coordinates are changed to spherical coordinates $q=\left(r \cos \theta_{1}, r \sin \theta_{1} \cos \theta_{2}, r \sin \theta_{1} \sin \theta_{2} \cos \theta_{3}, r \sin \theta_{1} \sin \theta_{2} \theta_{3}\right)$ where $\theta_{1}, \theta_{2} \in[0,2 \pi), \theta_{3} \in[0, \pi]$, and $z=x+y I$ for $q=x+I_{q} y$, where $x, y \in \mathbb{R}$.

$$
\begin{gathered}
M_{p}(r, f)^{p}=\frac{1}{4 \pi} \int_{\mathbb{S}(0, r)}\|f(q)\|^{p} d \mu \leq \frac{2^{p}}{4 \pi} \int_{\mathbb{S}(0, r)}\|f(z)\|^{p}+\|f(\bar{z})\|^{p} d \mu \\
\leq \frac{2^{p+1} \pi^{2}}{4 \pi} \int_{0}^{2 \pi}\left(\|f(z)\|^{p}+\|f(\bar{z})\|^{p}\right) d \theta_{1}=\frac{2^{p+2} \pi^{2}}{4 \pi} \int_{0}^{2 \pi}\|f(z)\|^{p} d \theta_{1} \\
\leq \frac{2^{2 p+2} \pi^{2}}{4 \pi} \int_{0}^{2 \pi}\left(\left\|f_{1}(z)\right\|^{p}+\left\|f_{2}(z)\right\|^{p}\right) d \theta_{1} \leq \frac{2^{2 p+2} \pi^{2}}{4 \pi} \int_{0}^{2 \pi}\left(\left\|g_{1}(z)\right\|^{p}+\left\|g_{2}(z)\right\|^{p}\right) d \theta_{1} \\
\leq \frac{2^{2 p+3} \pi^{2}}{4 \pi} \int_{0}^{2 \pi}\left(\|f(z)\|^{p}+\right) d \theta_{1}=2^{2 p+2} \pi^{2} M_{p, I}(r, g)
\end{gathered}
$$

Remark 4.12. We see that if $f, g \in \mathcal{R}(B(0 ; 1))$ with $f(0)=g(0)$, such that $g \prec_{\mathcal{N}} f$, then all inequalities of previous theorem are true for any $I \in \mathbb{S}$.

Theorem 4.13. (Rogosinski inequality) Let $f(q)=\sum_{k=1}^{\infty} q^{k} a_{k}, g(q)=$ $\sum_{k=1}^{\infty} q^{k} b_{k}$ with $f, g \in \mathcal{R}(B(0 ; 1)$. If $f \prec \mathcal{N} g$, then we have

$$
\sum_{k=1}^{n}\left\|a_{k}\right\|^{2} \leq \sum_{k=1}^{n}\left\|b_{k}\right\|^{2}, \text { for all } n=1,2, \ldots, .
$$

Proof. It is given in two cases:

(1) If $f, g \in \mathcal{V}(B(0 ; 1)$ ) (for $\mathcal{V}$ see the notation in Remark 3.12). From $f, g \in \mathcal{V}(B(0 ; 1))$, there exists $I, J \in \mathcal{S}$ such that $a_{k} \in \mathbb{C}_{I}$, and $b_{k} \in \mathbb{C}_{J}$, for all $k=1,2, \ldots$. From Definition 4.8, we have $f(q)=$ $g(w(q))$, with $w \in \mathcal{N}(B(0 ; 1))$, which immediately implies that $I=$ $J$. Therefore, it follows that $f, g \in \mathcal{V}_{I}(B(0 ; 1))$. Applying then Theorem 6.2, p. 192 in [16] (on the slice $\mathbb{C}_{I}$ ), we immediately get the required inequality.

(2) Consider $f, g \in \mathcal{R}(B(0 ; 1))$. Let $I, J \in \mathcal{S}$ and let $a_{1, k}, a_{2, k}, b_{1, k}, b_{2, k} \in$ $\mathbb{C}_{I}$ be such that $a_{k}=a_{1, k}+a_{2, k} J, b_{k}=b_{1, k}+b_{2, k} J$ for all $k=1,2, \ldots$, . Thus $f(q)=f_{1}(q)+f_{2}(q) J$ and $g(q)=g_{1}(q)+g_{2}(q) J$ for each $q \in B(0 ; 1)$, where

$$
f_{n}(q)=\sum_{k=1}^{\infty} q^{k} a_{n, k}, \quad \text { and } \quad g_{n}(q)=\sum_{k=1}^{\infty} q^{k} b_{n, k}, \quad n=1,2 .
$$


As $f \prec_{\mathcal{N}} g$ then $f_{n} \prec_{\mathcal{N}} g_{n}$ for $n=1,2$. From the previous case we have

$$
\sum_{k=1}^{n}\left\|a_{1, k}\right\|^{2} \leq \sum_{k=1}^{n}\left\|b_{1, k}\right\|^{2}, \quad \sum_{k=1}^{n}\left\|a_{2, k}\right\|^{2} \leq \sum_{k=1}^{n}\left\|b_{2, k}\right\|^{2} \text { for all } n=1,2, \ldots,
$$

and by adding respective terms one has the result.

The following result is on independent interest, but will also be useful later.

Proposition 4.14. Let $g: B(0 ; R) \rightarrow \mathbb{H}, R>0$, be a function slice regular of the form $g(q)=\sum_{n=0}^{\infty} q^{n} a_{n}$.

(1) There exists a power series $g_{r}^{-\bullet}(q)=\sum_{n=0}^{\infty} q^{n} b_{n}$ convergent in a disc with positive radius, such that $\left(g \bullet g_{r}^{-\bullet}\right)(q)=q$ and $g_{r}^{-\bullet}(0)=0$ if and only if $g(0)=0$ and $g^{\prime}(0) \neq 0$.

(2) There exists a power series $g_{l}^{-\bullet}(q)=\sum_{n=0}^{\infty} q^{n} b_{n}$ convergent in a disc with positive radius, such that $\left(g_{l}^{-\bullet} \bullet g\right)(q)=q$ and $g_{l}^{-\bullet}(0)=0$ if and only if $g(0)=0$ and $g^{\prime}(0) \neq 0$.

Proof. (1) Assume that $g_{r}^{-\bullet}$ exists. Then $\sum_{n=0}^{\infty}\left(\sum_{m=1}^{\infty} q^{m} b_{m}\right)^{* n} a_{n}=q$. By explicitly writing the terms of the equality we see that we have

$a_{0}+\left(\sum_{m=1}^{\infty} q^{m} b_{m}\right) a_{1}+\left(\sum_{m=1}^{\infty} q^{m} b_{m}\right)^{* 2} a_{2}+\ldots+\left(\sum_{m=1}^{\infty} q^{m} b_{m}\right)^{* n} a_{n}+\ldots=q$

and so to have equality it is necessary that $a_{0}=0$, i.e. $g(0)=0$, and $b_{1} a_{1}=1$ and so $a_{1} \neq 0$, i. e. $g^{\prime}(0) \neq 0$. To prove that the condition is sufficient, we observe that for $n \geq 2$, the coefficient of $q^{n}$ is zero on the right hand side of (4.5) while on the right hand side it is given by

$$
b_{n} a_{1}+P_{n}\left(b_{1}, \ldots, b_{n-1}, a_{2}, \ldots, a_{n}\right),
$$

thus we have $b_{n} a_{1}+P_{n}\left(b_{1}, \ldots, b_{n-1}, a_{2}, \ldots, a_{n}\right)=0$, (where the polynomials $P_{n}$ are linear in the $a_{i}$ 's and they contain all the possible monomials $b_{j_{1}} \ldots b_{j_{r}}$ with $j_{1}+\ldots+j_{r}=n$ and thus also $\left.b_{1}^{n}\right)$. In particular we have, $b_{1} a_{1}=1$ and so $b_{1}=a_{1}^{-1}, b_{2} a_{1}+b_{1}^{2} a_{2}=0$ and so $b_{2}=-a_{1}^{-2} a_{2} a_{1}^{-1}$. By induction, if we have computed $b_{1}, \ldots, b_{n-1}$ we can compute $b_{n}$ using (4.6) and the fact that $a_{1}$ is invertible and this concludes the proof. The function $g_{r}^{-\bullet}$ is right inverse of $g$, by its construction.

We now show that $g_{r}^{-\bullet}$ converges in a disc with positive radius following the proof of [7, Proposition 9.1]. Construct a power series with real coefficients $A_{n}$ which is a majorant of $g$ as follows: set $\bar{g}(q)=q A_{1}-\sum_{n=2}^{\infty} q^{n} A_{n}$ with $A_{1}=\left\|a_{1}\right\|$ and $A_{n} \geq\left\|a_{n}\right\|$, for all $n \geq 2$. It is possible to compute the inverse of $\bar{g}$ with respect to the (standard) composition to get the series $\bar{g}^{-1}(q)=\sum_{n=1} q^{n} B_{n}$. The coefficients $B_{n}$ can be computed with the formula

$$
B_{n} A_{1}+P_{n}\left(B_{1}, \ldots, B_{n-1}, A_{2}, \ldots, A_{n}\right),
$$


analogue of (4.6). Then we have $B_{1}=A_{1}^{-1}=\left\|a_{1}\right\|^{-1}, B_{2}=A_{1}^{-2}\left(-A_{2}\right) A_{1}^{-1} \geq$ $\left\|a_{1}\right\|^{-2}\left\|a_{2}\right\| \cdot\left\|a_{1}\right\|^{-1}=\left\|b_{2}\right\|$ and, inductively

$$
B_{n}=Q_{n}\left(A_{1}, \ldots, A_{n}\right) \geq Q_{n}\left(\left\|a_{1}\right\|, \ldots,\left\|a_{n}\right\|\right)=\left\|b_{n}\right\| .
$$

We conclude that the radius of convergence of $g_{r}^{-\bullet}$ is greater than or equal to the radius of convergence of $\bar{g}^{-1}$ which is positive, see [7, p. 27].

(2) It can be proven with computation similar to those used to prove (1). The function $g_{l}^{-\bullet}$ is left inverse of $g$.

Lemma 4.15. (1) If $g, w: B(0 ; 1) \rightarrow \mathbb{H}$ are slice regular functions and $g$ is univalent on $B(0 ; 1)$ with $g(0)=0$ and $g^{\prime}(0) \neq 0$, then $g_{l}^{-\bullet} \bullet$ $(g \bullet w)(q)=w(q)$, for all $q \in B(0 ; r)$ for a suitable $0<r<1$. If $w \in \mathcal{N}(B(0 ; 1)$ then $r=1$.

(2) If $g: B(0 ; 1) \rightarrow \mathbb{H}$ is a slice regular univalent function on $B(0 ; 1)$ with $g(0)=0, g^{\prime}(0) \neq 0$ and $w \in \mathcal{N}(B(0 ; 1))$, then $\partial_{s}(g \circ w)(0)=$ $\partial_{s}(g)(0) \cdot \partial_{s}(w)(0)$.

Proof. (1) The assertion follows from Proposition 4.6.

(2) We have:

$$
\partial_{s}(g \circ w)(0)=(g \circ w)^{\prime}(0)=\lim _{h \rightarrow 0} \frac{(g \circ w)(h)-(g \circ w)(0)}{h}, \quad h \in \mathbb{R} .
$$

Then the statement follows by standard arguments since we can write (remember that $w(0)=0)$ :

$$
\frac{(g \circ w)(h)-(g \circ w)(0)}{h}=\frac{g(w(h))-g(w(0))}{w(h)} \frac{w(h)}{h} .
$$

Proposition 4.16. If $f \prec_{\mathcal{N}} g$ then $f(0)=g(0)$ and $f(B(0 ; r)) \subseteq g(B(0 ; 1))$ for a suitable $0<r<1$.

Proof. By hypothesis, $f(q)=(g \circ w)(q))$ for all $q \in B(0 ; 1)$, where $w \in$ $\mathcal{N}(B(0 ; 1))$. By Schwarz's lemma (Theorem 4.1 in $[20]$ ), we get $\|w(q)\| \leq\|q\|$ for all $q \in B(0 ; 1)$, which implies $f(B(0 ; 1))=\{(g \circ w)(q) ; q \in B(0 ; 1)\}=$ $\{g(\xi) ;\|\xi\| \leq\|q\|, q \in B(0 ; 1)\} \subseteq g(B(0 ; 1))$.

Proposition 4.17. (1) If $f \prec \prec_{\mathcal{N}} g$ on $B(0 ; 1)$ then $f(\overline{B(0 ; r)}) \subset g(\overline{B(0 ; r)})$ for all $r \in(0,1)$, the equality taking place if and only if $f(q)=q \lambda$, with an $\lambda \in \mathbb{H}$ satisfying $\|\lambda\|=1$;

(2) If $f \prec_{\mathcal{N}}$ g on $B(0 ; 1)$ then $\max \{\|f(q)\| ;\|q\| \leq r\} \leq \max \{\|g(q)\| ;\|q\| \leq$ $r\}$ for all $r \in(0,1)$, the equality taking place if and only if $f(q)=q \lambda$, with an $\lambda \in \mathbb{H}$ satisfying $\|\lambda\|=1$;

(3) If $f \prec_{\mathcal{N}} g$ then $\left\|\partial_{s}(f)(0)\right\| \leq\left\|\partial_{s} g(0)\right\|$, the equality taking place if and only if $f(q)=q \lambda$, with an $\lambda \in \mathbb{H}$ satisfying $\|\lambda\|=1$.

Proof. The proof of (1), (2) follows from the Schwarz's lemma and reasoning as in the proof of Proposition 4.16.

(3) Since $w \in \mathcal{N}(B(0 ; 1))$, we get $\partial_{s}(f)(0)=\partial_{s}(g)(0) \cdot \partial_{s}(w)(0)$. Since by Schwarz's lemma we have $\left\|\partial_{s}(w)(0)\right\| \leq 1$, we get the desired conclusion. 


\section{Starlike and Convex Slice Regular Functions}

For our considerations, firstly we need the concepts of starlike and convex sets and functions.

Definition 5.1. (1) $A \subset \mathbb{H}$ is called starlike with respect to the origin 0 , if for all $t \in[0,1]$ we have $t A \subset A$.

(2) $A \subset \mathbb{H}$ is called convex if for all $t \in[0,1]$ we have $(1-t) A+t A \subset A$.

Remark 5.2. The definitions for starlike and convex sets in $\mathbb{H}$ are in fact the standard well-known definitions in $\mathbb{R}^{4}$. Thus, $A \subset \mathbb{R}^{4}$ with $0 \in A$, is starlike if for any point $p \in A$, the (Euclidean) segment determined by $p$ and the origin 0 is entirely contained in $A$. Also, the convexity of $A \subset \mathbb{R}^{4}$ is understood as the property that for any two points $p, q \in A$, the segment determined by $p$ and $q$ is entirely contained in $A$.

Definition 5.3. Let $f \in \mathcal{R}(B(0 ; 1))$ be a function satisfying $f(0)=0$ and $\partial_{s}(f)(0)=1$.

(1) $f$ is called slice-starlike on $B(0 ; 1)$ if for every $I \in \mathbb{S}$, we have

$$
\operatorname{Re}\left[(f(q))^{-1} \cdot q \cdot \partial_{s}(f)(q)\right]>0 \text {, for all } q=x+I y \in B(0 ; 1) \bigcap \mathbb{C}_{I} .
$$

(2) $f$ is called slice-convex on $B(0 ; 1)$ if for every $I \in \mathbb{S}$, we have

$$
\operatorname{Re}\left[\left(\partial_{s} f(q)\right)^{-1} \cdot q \cdot \partial_{s}^{2}(f)(q)\right]+1>0, \text { for all } q=x+I y \in B(0 ; 1) \bigcap \mathbb{C}_{I} .
$$

Remark 5.4. Taking into account the Splitting Lemma in Theorem 2.3, it is clear that in Definition 5.3 the corresponding inequalities characterize in fact the starlikeness and convexity of the $\mathbb{C}_{I}$-valued function $f_{I}: B(0 ; 1) \cap \mathbb{C}_{I} \rightarrow$ $\mathbb{C}_{I}$ of complex variable $z=x+I y$, where $f_{I}(z)=f(z)=f(x+I y)$, and $x^{2}+y^{2}<1$.

For more general classes of functions we introduce the following definition.

Definition 5.5. Let $f \in \mathcal{R}(B(0 ; 1))$ be a function satisfying $f(0)=0$ and $\partial_{s}(f)(0)=1$.

(1) $f$ is called starlike if $f(B(0 ; 1))$ is a starlike set with respect to the origin.

(2) $f$ is called convex if $f(B(0 ; 1)$ is a convex set.

(3) Let $I \in \mathbb{S}$, then $f$ is called $I$-starlike if $f\left(\mathbb{D}_{I}\right)$ is a starlike subset of $\mathbb{H}$.

(4) Let $I \in \mathbb{S}$, then $f$ is called $I$-convex if $f\left(\mathbb{D}_{I}\right)$ is a convex set.

Let $\operatorname{St}_{I}(B(0 ; 1))$ be the set of $I$-starlike slice regular functions on $B(0 ; 1)$.

Proposition 5.6. Let $J \in \mathbb{S}$ be any fixed element. Then

$\bigcap_{I \in \mathbb{S}} S t_{I}=\left\{f \in S t_{J} \mid t f(z)=f(w)\right.$ if and only if $\left.t f(\bar{z})=f(\bar{w}), z, w \in \mathbb{D}_{J}\right\}=: A_{J}$. 
Proof. The fact that $\bigcap_{I \in \mathbb{S}} S t_{I} \subseteq A_{J}$ follows from the definition of $A_{J}$. We prove that $A_{J} \subseteq \bigcap_{I \in \mathbb{S}} S t_{I}$. If $f \in A_{J}$ then for any $q=x+I_{q} y \in B(0 ; 1)$ the Representation Formula yields

$$
\begin{aligned}
& t f(q)=\frac{1}{2}\left[\left(1+I_{q} I\right) t f(\bar{z})+\left(1-I_{q} I\right) t f(z)\right] \\
& =\frac{1}{2}\left[\left(1+I_{q} I\right) f(\bar{w})+\left(1-I_{q} I\right) f(w)\right]=f(r),
\end{aligned}
$$

with $r=a+I_{q} b$.

Remark 5.7. Let $f \in S t_{I}$ and $J \in \mathbb{S}$ with $I \perp J$; let $f_{1}, f_{2} \in \operatorname{Hol}\left(\mathbb{D}_{I}\right)$ be such that $\left.f\right|_{D_{I}}=f_{1}+f_{2} J$. For any $z \in \mathbb{D}_{I}$ and any $t \in[0,1]$, there exists $w \in \mathbb{D}_{I}$ with

$$
t f(z)=f(w)
$$

which implies

$$
t f_{k}(z)=f_{k}(w)
$$

and so $f_{1}, f_{2}$ are starlike.

Theorem 5.8. Let $f \in \mathcal{N}(B(0 ; 1))$ be a function satisfying $f(0)=0$, $\partial_{s}(f)(0)=1$ and let $I \in \mathbb{S}$.

(1) Then, $f$ is $I$-convex if and only if $f$ is $J$-convex for any $J \in \mathbb{S}$.

(2) Then, $f$ is I-starlike if and only if $f$ is starlike.

Proof. Suppose that $f$ is $I$-convex. Then given any $q, v \in D_{J}$ with $q=$ $x+J y, v=a+J b$ and $x, y, a, b \in \mathbb{R}$, set $z=x+I y$ and $w=a+I b$. Then from the Representation Formula in Theorem 2.3 we have that $t f(q)+(1-t) f(v)$ is equal to

$$
\begin{aligned}
& \frac{1}{2}(1+J I) t f(\bar{z})+\frac{1}{2}(1-J I) t f(z)+\frac{1}{2}(1+J I)(1-t) f(\bar{w})+\frac{1}{2}(1-J I)(1-t) f(w) \\
& \quad=\frac{1}{2}(1+J I)(t f(\bar{z})+(1-t) f(\bar{w}))+\frac{1}{2}(1-J I)(t f(z)+(1-t) f(w)) \\
& =\frac{1}{2}(1+J I)(\overline{t f(z)+(1-t) f(w)})+\frac{1}{2}(1-J I)(t f(z)+(1-t) f(w)) \\
& =\frac{1}{2}(1+J I) \overline{f(u)}+\frac{1}{2}(1-J I) f(u)=\frac{1}{2}(1+J I) f(\bar{u})+\frac{1}{2}(1-J I) f(u)=f(s)
\end{aligned}
$$

where $s=c+J d$ if $u=c+I d \in \mathbb{D}_{I}$. We conclude that $f$ is $J$-convex. Exchanging the role of $I$ and $J$ we obtain the assertion.

To show (2), we assume that $f$ is $I$-starlike. Let $q \in B(0 ; 1)$, with $q=x+I_{q} y$, $x, y \in \mathbb{R}$ and $I_{q} \in \mathbb{S}$. Then

$$
\begin{gathered}
t f(q)=\frac{1}{2}(1+J I) t f(\bar{z})+\frac{1}{2}(1-J I) t f(z)=\frac{1}{2}(1+J I) \overline{t f(z)}+\frac{1}{2}(1-J I) t f(z) \\
=\frac{1}{2}(1+J I) f(\bar{u})+\frac{1}{2}(1-J I) f(u)=f(s) \in B(0 ; 1),
\end{gathered}
$$

where $s \in \mathbb{D}_{I_{q}}$, thus $f$ is starlike. The converse is trivially true. 
From the previous result and Remark 5.4 it follows that a function $\in \mathcal{N}(B(0 ; 1))$ can be simply called slice-convex instead of $I$-convex.

Let $I \in \mathbb{S}$, denote

$$
\mathcal{P}_{I}=\left\{f \in \mathcal{R}(B(0 ; 1)) \mid \operatorname{Re}(f(z))>0, z \in \mathbb{D}_{I}, f(0)=1\right\} .
$$

Theorem 5.9. Let $f \in \mathcal{N}(B(0 ; 1))$ be such that $f(0)=0$ and $\partial_{s} f(0)=1$.

(1) Then $f$ is a starlike function if and only if the function $q \partial_{s} f(q) *$ $f(q)^{-*}, \quad q \in B(0 ; 1)$ belongs to $\mathcal{P}_{I}$ for each $I \in \mathbb{S}$.

(2) Let $I \in \mathbb{S}$, then $f$ is I-convex function if and only if the function $1+q \partial_{s}^{2} f(q) *\left(\partial_{s} f(q)\right)^{-*}, \quad q \in B(0 ; 1)$ belongs to $\mathcal{P}_{I}$.

(3) Let $I \in \mathbb{S}$, then $f$ is a $I$-convex function if and only if the function $q * \partial_{s} f(q), \quad q \in B(0 ; 1)$ is a I-starlike function.

(4) If $f$ is a starlike function, then its coefficients satisfy $\left\|a_{n}\right\| \leq n$ for $n \in \mathbb{N}$. Strict inequality holds for all $n$ unless $f$ is a rotation of the Koebe function.

(5) Let $I \in \mathbb{S}$. If $f$ is a I-convex function, then $\left\|a_{n}\right\| \leq 1$ for $n=$ $2,3, \ldots$ Strict inequality holds for all $n$ unless $f$ is a rotation of $q(1-q)^{-1}$.

(6) Let $I \in \mathbb{S}$. If $f$ is a $I$-convex function then $B(0 ; 1 / 2) \cap \mathbb{C}_{I} \subset$ $f\left[B(0 ; 1) \cap \mathbb{C}_{I}\right]$.

Proof. Since $f \in \mathcal{N}(B(0 ; 1))$, we have that $f: B(0 ; 1) \cap \mathbb{C}_{I} \rightarrow \mathbb{C}_{I}$, is a holomorphic function on $B(0 ; 1) \cap \mathbb{C}_{I}$ for every $I \in \mathbb{S}$.

1. According to the above Theorem 5.8, point 2 and by Theorem 2.10 in [16] (applied on every slice $\mathbb{C}_{I}$ ), we get the desired conclusion.

2. It is immediate by Theorem 2.11 in [16] applied on the given slice $\mathbb{C}_{I}$.

3. It is immediate by Theorem 2.12 in [16] (Alexander's theorem) applied on the given slice $\mathbb{C}_{I}$.

4. It is immediate by the above Theorem 5.8, point 2 and by Theorem 2.14 in [16] applied on any fixed slice $\mathbb{C}_{I}$.

5. It immediate from the above Theorem 5.8, point 1 , and the Corollary from the page 45 in [16] applied on the slice $\mathbb{C}_{I}$.

6. It is immediate from Theorem 2.15 in [16] applied on the slice $\mathbb{C}_{I}$.

We can prove the following geometric characterizations of the slice-starlike and slice-convex functions.

Theorem 5.10. Let $f \in \mathcal{N}(B(0 ; 1))$ be a function satisfying $f(0)=0$ and $\partial_{s}(f)(0)=1$. If $f$ is slice-starlike on $B(0 ; 1)$ then $f$ is univalent in $B(0 ; 1)$ and $f[B(0 ; 1)]$ is a starlike set. Moreover, denoting $B(0 ; r)=\{q \in \mathbb{H} ;\|q\|<$ $r$, also $f[B(0 ; r)]$ is a starlike set for every $0<r<1$.

Proof. Firstly, we will prove that $f$ is univalent in $B(0 ; 1)$. In this sense, we have four cases.

Case 1). For a fixed $I \in \mathbb{S}$, let $q_{1}, q_{2} \in B(0 ; 1) \cap \mathbb{C}_{I}$ be with $q_{1} \neq q_{2}$. By the Remark 5.4 and by the starlikeness of $f$, it follows (see e.g. [26], p. 45-46 or [28]) the univalence of $f$ on $B(0 ; 1) \cap \mathbb{C}_{I}$, that is $f\left(q_{1}\right) \neq f\left(q_{2}\right)$. 
Case 2). Let $q_{1}=a_{1} \in \mathbb{R}, q_{2}=a_{2} \in \mathbb{R}$ be with $q_{1} \neq q_{2}$. Choosing an arbitrary $I \in \mathbb{S}$ we can write $q_{1}=a_{1}+I \cdot 0, q_{2}=a_{2}+I \cdot 0$ and we are in Case 1), obtaining thus $f\left(q_{1}\right) \neq f\left(q_{2}\right)$.

Case 3). Let $q_{1} \in \mathbb{C}_{I}, q_{2} \in \mathbb{C}_{-I}$ be with $q_{1} \neq q_{2}$. But it is easy to see that $\mathbb{C}_{I}=\mathbb{C}_{-I}$, which means that we are in the Case 1) and we obtain $f\left(q_{1}\right) \neq f\left(q_{2}\right)$.

Case 4). Let $q_{1} \in B(0 ; 1) \cap \mathbb{C}_{J}$ and $q_{2} \in B(0 ; 1) \cap \mathbb{C}_{I}$ be with $q_{1} \neq q_{2}$, where $I, J \in \mathbb{S}, J \neq I$ and $J \neq-I$. Since by Theorem 2.3 , (ii) we have $f\left(q_{1}\right)=a+J b$ and $f\left(q_{2}\right)=c+I d$, if we would have $f\left(q_{1}\right)=f\left(q_{2}\right)$, then we would necessarily get two subcases : (i) $a=c$ and $b=d=0$ or (ii) $a=c$ and $I=J, b=c \neq 0$.

Subcase $\left(4_{i}\right)$. It follows that $q_{1}, q_{2} \in \mathbb{R}$, which according to the Case 2) would imply $f\left(q_{1}\right) \neq f\left(q_{2}\right)$, a contradiction.

Subcase $\left(4_{i i}\right)$. This is impossible because it implies $I=J$, a contradiction.

Collecting the above results, it follows the univalence of $f$ in $B(0 ; 1)$.

Now we prove that $f(B(0 ; 1))$ is starlike. Writing $\mathbb{D}_{I}=B(0 ; 1) \bigcap \mathbb{C}_{I}$, we have $B(0 ; 1)=\bigcup_{I \in \mathbb{S}} \mathbb{D}_{I}, f(B(0 ; 1))=f\left(\bigcup_{I \in \mathbb{S}} \mathbb{D}_{I}\right)=\bigcup_{I \in \mathbb{S}} f\left(\mathbb{D}_{I}\right)$, which implies for all $t \in[0,1]$,

$$
t f(B(0 ; 1))=\bigcup_{I \in \mathbb{S}} t f\left(\mathbb{D}_{I}\right) \subset \bigcup_{I \in \mathbb{S}} f\left(\mathbb{D}_{I}\right)=f(B(0 ; 1)) .
$$

We used here the fact that the starlikeness of $f: \mathbb{D}_{I} \rightarrow \mathbb{C}_{I}$ as function of $z=x+I y \in \mathbb{D}_{I}$ implies that the set $f\left(\mathbb{D}_{I}\right)$ is starlike (see e.g. [26], p. 44-45).

Similarly, denoting $\mathbb{D}_{I}(0 ; r)=B(0 ; r) \cap \mathbb{C}_{I}$ and taking into account that the starlikeness of $f: \mathbb{D}_{I}(0 ; 1) \rightarrow \mathbb{C}_{I}$ as function of $z=x+I y \in \mathbb{D}_{I}$ also implies (see again e.g. [26], p. 44-45) that $f\left(\mathbb{D}_{I}\right)$ is starlike, reasoning as above we immediately obtain that $f(B(0 ; r))$ is starlike, for every $r \in$ $(0,1)$.

Remark 5.11. The geometric properties in Theorem 5.10 are similar to those obtained in the case of a complex variable. Also, note that from the proof of Theorem 5.10 it easily follows that if $f \in \mathcal{N}(B(0 ; 1))$ is a function satisfying $f(0)=0, \partial_{s}(f)(0)=1$, such that $f(z)$ with $z \in \mathbb{C}$ is univalent in the open unit disk in $\mathbb{C}$, then as function of $q, f$ is univalent in $B(0 ; 1)$.

Theorem 5.12. Let $f \in \mathcal{N}(B(0 ; 1))$ be a function satisfying $f(0)=0$ and $\partial_{s}(f)(0)=1$. If $f$ is slice-convex on $B(0 ; 1)$ then $f$ is univalent in $B(0 ; 1)$ and $f[B(0 ; 1)]$ is a convex set. Moreover, denoting $B(0 ; r)=\{q \in \mathbb{H} ;\|q\|<$ $r\}$, also $f[B(0 ; r)]$ is a convex set for every $0<r<1$.

Proof. The univalence of $f$ in $B(0 ; 1)$ follows exactly as in the proof of Theorem 5.10, by taking into account that the convexity of a function of complex variable implies its univalence (see e.g. [26], p. 51 or [28]).

We now prove the convexity of $f[B(0 ; 1)]$ and of $f[B(0 ; r)]$ for every $0<$ $r<1$. First recall that quaternionic intrinsic functions take any slice to itself, i.e. $f\left(\mathbb{D}_{I}\right) \subset \mathbb{C}_{I}$. Then we use the relation obtained in the proof of 
Theorem 5.10, $f[B(0 ; 1)]=\bigcup_{I \in \mathbb{S}} f\left(\mathbb{D}_{I}\right)$, where every $f\left(\mathbb{D}_{I}\right)$ is convex from the hypothesis and from [26], p. 49-50 (that is $t f\left(\mathbb{D}_{I}\right)+(1-t) f\left(\mathbb{D}_{I}\right) \subset f\left(\mathbb{D}_{I}\right)$, for all $t \in[0,1])$. Moreover for any quaternion $x+I y \in B(0,1)$, we have that if $f(x+I y)=u+I v \in f[B(0,1)]$ then also $u+\tilde{I} v \in f[B(0,1)]$ for any other $\tilde{I} \in \mathbb{S}$, in fact $u+\tilde{I} v=f(x+\tilde{I} v)$ and $x+\tilde{I} y$ obviously belongs to $B(0,1)$.

We have to show that for any two points $f\left(q_{1}\right), f\left(q_{2}\right) \in f[B(0 ; 1)]$ we have $t f\left(q_{1}\right)+(1-t) f\left(q_{2}\right) \in f[B(0 ; 1)]$ for any $t \in[0,1]$. We take $q_{1}=x_{1}+I y_{1}$, $q_{2}=x_{2}+L y_{2}$ and $f\left(q_{1}\right)=u_{1}+I v_{1}, f\left(q_{2}\right)=u_{2}+L v_{2}$. Take $J \in \mathbb{S}$ such that $I, J, I J=K$ is a basis of $\mathbb{H}$ and rewrite $L=\alpha_{1} I+\alpha_{2} J+\alpha_{3} K$, with $\sum_{i=1}^{3} \alpha_{i}^{2}=1$ and

$$
\begin{aligned}
t f\left(q_{1}\right)+(1-t) f\left(q_{2}\right) & =t\left(u_{1}+I v_{1}\right)+(1-t)\left(u_{2}+\left(\alpha_{1} I+\alpha_{2} J+\alpha_{3} K\right) v_{2}\right) \\
& =\left(t u_{1}+(1-t) u_{2}\right)+\tilde{I} v\left(t, v_{1}, v_{2}, \alpha_{1}\right)
\end{aligned}
$$

where we set

$$
w=\left(t v_{1}+(1-t) \alpha_{1} v_{2}\right) I+(1-t)\left(\alpha_{2} v_{2} J+\alpha_{3} v_{2} K\right), \quad \tilde{I}:=\frac{w}{\|w\|}
$$

and

$$
\begin{aligned}
v\left(t, v_{1}, v_{2}, \alpha_{1}\right) & =\left\|\left(t v_{1}+(1-t) \alpha_{1} v_{2}\right) I+(1-t)\left(\alpha_{2} v_{2} J+\alpha_{3} v_{2} K\right)\right\| \\
& =\left(t^{2} v_{1}^{2}+(1-t)^{2} v_{2}^{2}+2 t(1-t) \alpha_{1} v_{1} v_{2}\right)^{1 / 2} .
\end{aligned}
$$

To show that $\left(t u_{1}+(1-t) u_{2}\right)+\tilde{I} v\left(t, v_{1}, v_{2}, \alpha_{1}\right)$ belongs to $f(B(0 ; 1))$ we first observe that $\left|v\left(t, v_{1}, v_{2}, \alpha_{1}\right)\right| \leq\left|t v_{1}+(1-t) v_{2}\right|$. The segment joining any point of the form $t f\left(q_{1}^{\prime}\right)+(1-t) f\left(q_{2}^{\prime}\right)$, for any $t \in[0,1]$ fixed, with its conjugate $\overline{t f\left(q_{1}^{\prime}\right)+(1-t) f\left(q_{2}^{\prime}\right)}=t f\left(\bar{q}_{1}^{\prime}\right)+(1-t) f\left(\bar{q}_{2}^{\prime}\right)$, where $q_{i}^{\prime}=x_{i}+\tilde{I} y_{i}$, $i=1,2$ belongs to $f\left(\mathbb{D}_{\tilde{I}}\right)$, and so to $f(B(0 ; 1))$, by its convexity and so also the point $\left(t u_{1}+(1-t) u_{2}\right)+\tilde{I} v\left(t, v_{1}, v_{2}, \alpha_{1}\right)$ belongs to $f\left(\mathbb{D}_{\tilde{I}}\right)$ since it belongs to that segment.

Indeed, denoting $t f\left(q_{1}^{\prime}\right)+(1-t) f\left(q_{2}^{\prime}\right)=A+\tilde{I} B$, we have

$$
\overline{t f\left(q_{1}^{\prime}\right)+(1-t) f\left(q_{2}^{\prime}\right)}=A-\tilde{I} B
$$

and the segment $S=\left[t f\left(q_{1}^{\prime}\right)+(1-t) f\left(q_{2}^{\prime}\right), \overline{t f\left(q_{1}^{\prime}\right)+(1-t) f\left(q_{2}^{\prime}\right)}\right]$ can be written as the set

$$
\begin{aligned}
S & =\{\lambda(A+\tilde{I} B)+(1-\lambda)(A-\tilde{I} B) ; \lambda \in[0,1]\} \\
& =\{A+\tilde{I}[\lambda B+(1-\lambda)(-B)] ; \lambda \in[0,1]\} .
\end{aligned}
$$

On the other hand, denoting $f\left(q_{1}^{\prime}\right)=u_{1}+\tilde{I} v_{1}$ and $f\left(q_{2}^{\prime}\right)=u_{2}+\tilde{I} v_{2}$ (since $f$ is intrinsic function), for fixed $t \in[0,1]$ we get

$$
\begin{aligned}
& A+\tilde{I} B=t u_{1}+(1-t) u_{2}+\tilde{I}\left(t v_{1}+(1-t) v_{2}\right), \\
& A-\tilde{I} B=t u_{1}+(1-t) u_{2}-\tilde{I}\left(t v_{1}+(1-t) v_{2}\right)
\end{aligned}
$$

and by (5.1) the segment $S$ can be written as $S=\left\{t u_{1}+(1-t) u_{2}+\tilde{I}\left[\lambda\left(t v_{1}+(1-t) v_{2}\right)+(1-\lambda)\left(-t v_{1}-(1-t) v_{2}\right)\right] ; \lambda \in[0,1]\right\}$. 
Now, since $\left|v\left(t, v_{1}, v_{2}, \alpha_{1}\right)\right| \leq\left|t v_{1}+(1-t) v_{2}\right|$, it follows that there exists a $\lambda \in[0,1]$ such that

$$
v\left(t, v_{1}, v_{2}, \alpha_{1}\right)=\lambda\left(t v_{1}+(1-t) v_{2}\right)+(1-\lambda)\left(-t v_{1}-(1-t) v_{2}\right),
$$

which implies that $\left(t u_{1}+(1-t) u_{2}\right)+\tilde{I} v\left(t, v_{1}, v_{2}, \alpha_{1}\right)$ belongs to the segment $S$.

The convexity of $f[B(0, r)], 0<r<1$ can be proven similarly.

Remark 5.13. The geometric properties in Theorem 5.12 are similar to those obtained in the case of a complex variable.

Remark 5.14. As consequences/applications of Theorem 5.10 and 5.12, we can easily generate many examples of transformations from $\mathbb{R}^{4}$ to $\mathbb{R}^{4}$, with nice geometric properties, because any $f: B(0,1) \rightarrow \mathbb{H}$ can be written in the form (3.3) and therefore $f$ can be also be viewed as the transformation

$$
\begin{gathered}
\left(x_{1}, x_{2}, x_{3}, x_{4}\right) \rightarrow \\
\left(P_{1}\left(x_{1}, x_{2}, x_{3}, x_{4}\right), P_{2}\left(x_{1}, x_{2}, x_{3}, x_{4}\right), P_{3}\left(x_{1}, x_{2}, x_{3}, x_{4}\right), P_{4}\left(x_{1}, x_{2}, x_{3}, x_{4}\right)\right),
\end{gathered}
$$

with all $P_{k}\left(x_{1}, x_{2}, x_{3}, x_{4}\right), k=1,2,3,4$, real-valued. Thus, if in the expression of an analytic function of complex variable with the coefficients in the series expansions all real, which is a starlike (or convex) function, we replace $z$-complex, by $q$-quaternion, we get in fact a (infinite differentiable) mapping from $\mathbb{R}^{4}$ to $\mathbb{R}^{4}$, which transforms the Euclidean open unit ball and all its open sub-balls (that is with the same center at origin) into starlike (respectively convex) bodies in $\mathbb{R}^{4}$.

Just to get a flavor, we present here a few concrete examples.

Example 3. (1) Polynomials with real coefficients, like :

a) $f(q)=q[1-q /(n+1)]^{n-1}, q$-quaternion, $n \in \mathbb{N}$ arbitrary, is univalent and transforms the Euclidean open unit ball in $\mathbb{R}^{4}$ and all its open sub-balls, into starlike bodies in $\mathbb{R}^{4}$ (because $f(z), z \in \mathbb{C}$ is starlike, see e.g. 22 );

b) $f(q)=q+a_{2} q^{2} /\left(2^{2}\right)+\ldots+a_{n} q^{n} /\left(n^{2}\right), n \in \mathbb{N}$ arbitrary, $q-$ quaternion, where all $a_{k}$ are real and satisfy the inequality $1 \geq$ $\sum_{k=2}^{n}\left|a_{k}\right|$, is univalent and transforms the Euclidean open unit ball in $\mathbb{R}^{4}$ and all its open sub-balls, into convex bodies in $\mathbb{R}^{4}$ (because $f(z), z \in \mathbb{C}$ is convex, see e.g. [1]);

(2) If we define the elementary functions of quaternion variable, exp, sin, cos and log simply replacing in the expressions of their series expansions in $\mathbb{C}, z \in \mathbb{C}$ by $q$-quaternion, then we get non-polynomial transformations with nice geometric properties, like, for example, the following :

a) $f(q)=(1-\lambda) q+\lambda \sin (q)$, $q$-quaternion, is univalent and transform the Euclidean ball in $\mathbb{R}^{4}$ and all its open sub-balls, into convex bodies in $\mathbb{R}^{4}$, if $\lambda \in \mathbb{R}$ and satisfies the inequality $|\lambda| \leq 4 e /\left[3\left(e^{2}-1\right)\right]$ (because $f(z), z \in \mathbb{C}$ is convex, see e.g. [25]); 
b) $f(q)=q+\lambda\left[\exp (q)-1-q-q^{2} / 2\right]$, is univalent and transforms the Euclidean ball in $\mathbb{R}^{4}$ and all its open sub-balls, into convex bodies in $\mathbb{R}^{4}$, if $\lambda \in \mathbb{R}$ and satisfies the inequality $|\lambda| \leq 2 /[3(e-1)]$ (because $f(z), z \in \mathbb{C}$ is starlike, see e.g. 25]), and into starlike bodies in $\mathbb{R}^{4}$, if $\lambda \in \mathbb{R}$ and satisfies the inequality $|\lambda| \leq 3 /[(e-2) \sqrt{10}]$ (because $f(z), z \in \mathbb{C}$ is convex in this case, see e.g. [25]).

(3) The Koebe function $K(q), q \in \mathbb{H}$, is univalent and transforms the Euclidean open unit ball in $\mathbb{R}^{4}$ and all its open sub-balls, into starlike bodies in $\mathbb{R}^{4}$. This is because $f(z)=z \cdot \frac{1}{(1-z)^{2}}=z+2 z^{2}+3 z^{3}+\ldots$, with $|z|<1$, is an analytic starlike function.

Remark 5.15. It is worth noting that the above examples of univalent, starlike and convex functions of quaternion variable, are strongly contrasting with what happens in the case of geometric function theory for functions of several complex variables, when it is surprisingly difficult to construct starlike or convex mappings for analytic functions of several complex variables defined in the Euclidean open unit ball, by starting from starlike and convex functions of one complex variable (see e.g. [23], Subsection 6.3.3 and Problems 6.3.2, 6.3.3,(i)).

As in the case of complex variable, we have the following result.

Proposition 5.16. A normalized function $f \in \mathcal{R}(B(0 ; 1))$ is slice-convex if and only if the function $h(q)=q \cdot \partial_{s}(f)(q)$ is slice-starlike.

Proof. This easily follows from the fact that in Definition 5.3, the inequality in the point 2 for $f$ is equivalent to the inequality in the point 1 for $h(q)=$ $q \cdot \partial_{s}(f)(q)$. Indeed, we can write

$$
\begin{gathered}
(h(q))^{-1} \cdot q \cdot \partial_{s}(h)(q)=(h(q))^{-1} \cdot q \cdot\left[\partial_{s}(f)(q)+q \cdot \partial_{s}^{2}(f)(q)\right] \\
=\left(\partial_{s}(f)(q)\right)^{-1} \cdot q^{-1} \cdot q \cdot\left[\partial_{s}(f)(q)+q \cdot \partial_{s}^{2}(f)(q)\right]=1+\left(\partial_{s}(f)(q)\right)^{-1} \cdot q \cdot \partial_{s}^{2}(f)(q),
\end{gathered}
$$
which proves the assertion.

Remark 5.17. Let $f(q)=q+\sum_{k=2}^{\infty} q^{k} a_{k}$ (with $a_{k} \in \mathbb{H}, k=0,1, \ldots$ ) be slice regular and satisfying $f(0)=0$ and $\partial_{s}(f)(0)=1$. Consider the Alexanderkind operator $A(f)(q)=\int_{0}^{q} t^{-1} \cdot f(t) d t$ and the Libera operator $L(f)(q)=$ $2 q^{-1} \int_{0}^{q} f(t) d t$, defined for $q \in B(0 ; 1)$.

Firstly we get $A(f)(q)=q+\sum_{k=2}^{\infty} \frac{1}{k^{2}} q^{k} a_{k}$. Since $q \cdot \partial_{s}[A(f)(q)]=f(q)$ for all $q \in B(0 ; 1)$, by Lemma 5.16 it follows that $f$ is slice-starlike if and only if the Alexander integral operator $A(f)$ is slice-convex on $B(0 ; 1)$. In other words, the Alexander integral operator transforms a slice starlike function into a slice convex function on $B(0 ; 1)$

Secondly, we get $L(f)(q)=2 q^{-1} \int_{0}^{q} f(t) d t=q+2 \sum_{k=2}^{\infty} \frac{q^{k}}{k+1} \cdot a_{k}$. Now, suppose that $f$ is slice-starlike in $B(0 ; 1)$ and that $f \in \mathcal{N}(B(0 ; 1))$. It follows that the coefficients $a_{k} \in \mathbb{R}$, for all $k \geq 2$ and then since $f(z)$ in starlike on the open unit disk of the complex plane, by the result in 24 we get that $L(f)(z)$ is starlike in the open unit disk of the complex plane. Taking into 
account Theorem [5.10, it follows that $L(f)(q)$ is slice starlike in the ball $B(0 ; 1)$.

\section{Spirallike Slice Regular Functions}

In what follows, we deal with spirallike functions of quaternion variable. In this sense, firstly we introduce the following well-known concept.

Definition 6.1. (see e.g. 23, Remark 6.4.11) If $X$ is a linear space over $\mathbb{C}$, then $A \subset X$ is called spirallike of type $\gamma \in\left(-\frac{\pi}{2}, \frac{\pi}{2}\right)$, if for all $w \in A$ and all $t \geq 0$ we have $e^{-t \lambda} w \in A$, where $\lambda=e^{-i \gamma}$.

Remark 6.2. The curve in $X$ of equation $s(t)=e^{-t \lambda} w_{0}$, with $\lambda=e^{-i \gamma}$ and $w_{0} \in X$ fixed, is called $\gamma$-spiral in $X$ that joins $w_{0}$ with the origin. Therefore, $A \subset X$ is $\gamma$-spirallike if for any point $w_{0} \in A$, the $\gamma$-spiral that joins $w_{0}$ and the origin, entirely belongs to $A$. When $X=\mathbb{C}$ then $s(t), t \geq 0$ becomes the well-known logarithmic spiral in the plane that joins $w_{0}$ with the origin.

Remark 6.3. Since $\mathbb{H}$ obviously is a linear space over $\mathbb{C}$, the parametric equations of the $\gamma$-spiral in $\mathbb{H}, s(t)=e^{-t \lambda} q_{0}$, where $\lambda=e^{-i \gamma}$ and $q_{0}=$ $x_{0}+i y_{0}+j z_{0}+k u_{0} \in \mathbb{H}$, can easily derived by simple calculation as

$$
\begin{gathered}
x(t)=A(t)\left\{\cos [B(t)] x_{0}-\sin [B(t)] y_{0}\right\}, y(t)=A(t)\left\{\cos [B(t)] y_{0}+\sin [B(t)] x_{0}\right\}, \\
x(t)=A(t)\left\{\cos [B(t)] z_{0}-\sin [B(t)] u_{0}\right\}, y(t)=A(t)\left\{\cos [B(t)] u_{0}+\sin [B(t)] z_{0}\right\}, \\
t \geq 0,
\end{gathered}
$$

where $A(t)=e^{-t \cos (\gamma)}$ and $B(t)=t \sin (\gamma)$.

Now, we are in position to introduce the concept of spirallike function.

Definition 6.4. Let $f \in \mathcal{R}(B(0 ; 1))$ be a function satisfying $f(0)=0$ and $\partial_{s}(f)(0)=1$. Also, let $\gamma \in\left(\frac{-\pi}{2}, \frac{\pi}{2}\right)$.

Then, $f$ is called slice-spirallike of $\gamma$-type on $B(0 ; 1)$, if for every $I \in \mathbb{S}$, we have

$$
\operatorname{Re}\left[e^{-I \gamma} \cdot q \cdot \partial_{s}(f)(q) \cdot \frac{1}{f(q)}\right]>0, \text { for all } q=x+I y \in B(0 ; 1) \bigcap \mathbb{C}_{I},
$$

where $e^{-I \gamma}=\cos (\gamma)-I \sin (\gamma)$.

Remark 6.5. Evidently that for $\gamma=0$, we recapture the concept of slicestarlike function in Definition 5.3, (i).

We have:

Theorem 6.6. Let $f \in \mathcal{N}(B(0 ; 1))$ be a function satisfying $f(0)=0$ and $\partial_{s}(f)(0)=1$ and let $\gamma \in\left(\frac{-\pi}{2}, \frac{\pi}{2}\right)$. If $f$ is slice-spirallike of type $\gamma$ on $B(0 ; 1)$ then $f$ is univalent in $B(0 ; 1)$ and $f[B(0 ; 1)]$ is a spirallike set of $\gamma$-type. 
Proof. The univalence of $f$ in $B(0 ; 1)$ follows exactly as in the proof of Theorem 5.10, by taking into account that the spirallikeness of $\gamma$-type of a function of complex variable implies its univalence.

It was remained to prove the spirallikeness of $\gamma$-type of $f[B(0 ; 1)]$. With the notations in the proofs of Theorems 5.10 and 5.12 , we have $B(0 ; 1)=$ $\bigcup_{I \in \mathbb{S}} \mathbb{D}_{I}$ and $f[B(0 ; 1)]=\bigcup_{I \in \mathbb{S}} f\left(\mathbb{D}_{I}\right)$, where every $f\left(\mathbb{D}_{I}\right)$ is $\gamma$-spirallike according to Definition 6.4 and by [26], p. 42, Theorem 4.4.1.

Taking $e^{-\lambda t}$ with $\lambda=e^{-i \gamma}$, this implies, for all $t \geq 0$,

$$
e^{-\lambda t} f[B(0 ; 1)]=\bigcup_{I \in \mathbb{S}} e^{-\lambda t} f\left(\mathbb{D}_{I}\right) \subset \bigcup_{I \in \mathbb{S}} f\left(\mathbb{D}_{I}\right)=f(B(0 ; 1)),
$$

which proves the theorem.

Remark 6.7. As in the cases of starlikeness and convexity, we can easily construct spirallike functions of quaternion variable, from spirallike functions of complex variable whose series expansions have all coefficients real, simply by replacing into their expression $z \in \mathbb{C}$, by $q$-quaternion.

Open question. An interesting question would be to find large subclasses of functions in $\mathcal{R}(B(0 ; 1))$ different from the class $\mathcal{N}(B(0 ; 1))$, defined as in Definitions 5.3 and 6.4, for which the univalence and the geometric properties in Theorems [5.10, 5.12 and 6.6 still hold. As some very particular cases, for example when $f$ is of the form $f(q)=h(q) \cdot C_{0}, q \in B(0 ; 1)$, with $h \in \mathcal{N}(B(0 ; 1))$ and $C_{0} \in \mathbb{H}$ a constant, clearly $f \notin \mathcal{N}(B(0 ; 1))$ and if $h$ satisfies one of the Definitions [5.3. (i), (ii), or 6.4, then it is easy to see that $f$ also satisfies the same kind of definition. At the same time, since $h$ has one of the corresponding geometric property (including univalence) in Theorems 5.10, 5.12 and 6.6. it is easy to check directly that $f=h \cdot C_{0}$ keeps the same geometric property (and univalence) of $h$.

\section{REFERENCES}

[1] J.W. Alexander, Functions which map the interior of the unit circle upon simple regions, Ann. of Math., 17(1915), 12-22.

[2] D. Alpay, V. Bolotnikov, F. Colombo, I. Sabadini, Self-mappings of the quaternionic unit ball: multiplier properties, Schwarz-Pick inequality, and Nevanlinna-Pick interpolation problem, To appear in the Indiana Mathematical Journal of Mathematics.

[3] D. Alpay, F. Colombo, D. P. Kimsey, I. Sabadini, An extension of Herglotz's theorem to the quaternions, J. Math. Anal. Appl. (2015).

[4] D. Alpay, F. Colombo, and I. Sabadini, Schur functions and their realizations in the slice hyperholomorphic setting, Integral Equations and Operator Theory, 72 (2012), 253-289.

[5] D. Alpay, F. Colombo, and I. Sabadini, Pontryagin-de Branges-Rovnyak spaces of slice hyperholomorphic functions, J. Anal. Math., 121 (2013), 87-125.

[6] C. Bisi, C. Stoppato, The Schwarz-Pick lemma for slice regular functions, Indiana Univ. Math. J. 61 (2012), no. 1, 297-317.

[7] H. Cartan, Elementary Theory of Analytic Functions of One or Several Complex Variables, Dover Publ. Inc., New York, 1995.

[8] F. Colombo, I. Sabadini, Some remarks on the $\mathcal{S}$-spectrum, Compl. Var. Ell. Eq., 58(2013), no. 1, 1-6. 
[9] F. Colombo, J. Oscar González-Cervantes, I. Sabadini, On slice biregular functions and isomorphisms of Bergman spaces, Compl. Var. Ell. Eq., 57(2012), nos. 78, 825839.

[10] F. Colombo, J. Oscar González-Cervantes, I. Sabadini, The C-property for slice regular functions and applications to the Bergman space, Compl. Var. Ell. Eq., 58(2013), no. 10, 1355-1372.

[11] F. Colombo, J. Oscar González-Cervantes, I. Sabadini, A nonconstant coefficients differential operator associated to slice monogenic functions, Trans. Amer. Math. Soc. 365 (2013), 303-318.

[12] F. Colombo, I. Sabadini, D.C. Struppa, Noncommutative Functional Calculus. Theory and Applications of Slice Hyperholomorphic Functions, Birkhauser, Basel, 2011.

[13] L. de Branges, A proof of Bieberbach conjecture, Acta Math., 154(1985), 137-152.

[14] C. Della Rocchetta, G. Gentili, G. Sarfatti, A Bloch-Landau theorem for slice regular functions, Advances in hypercomplex analysis, Springer INdAM Ser.,1, 55-74, Springer, Milan, 2013.

[15] C. Della Rocchetta, G. Gentili, G. Sarfatti, The Bohr theorem for slice regular functions, Math. Nachr., 285 (2012), 2093-2105.

[16] P. L. Duren, Univalent Functions, Springer-Verlag, New York - Berlin - Heidelberg Tokyo, 1983.

[17] S.G. Gal, Introduction to Geometric Function Theory of Hypercomplex Variables, Nova Science Publishers, Inc., Hauppauge, New York, 2002.

[18] G. Gentili, G. Sarfatti, Landau-Toeplitz theorems for slice regular functions over quaternions, Pacific J. Math., 265 (2013), 381-404.

[19] G. Gentili, C Stoppato, D.C. Struppa, Regular functions of a quaternionic variable, Springer Monographs in Mathematics, Springer, Berlin-Heidelberg (2013).

[20] G. Gentili, D.C. Struppa, A new theory of regular functions of a quaternion variable, Advances in Mathematics, 216(2007), 279-301.

[21] R. Ghiloni, A. Perotti, Slice regular functions on real alternative algebras, Adv. Math., 226 (2011), 1662-1691.

[22] A. Gluchoff, F. Hartmann, Starlikeness of polynomials and finite Blaschke products, Ann. Polon. Math., 93(2008), no. 2, 187-196.

[23] I. Graham, G. Kohr, Geometric Function Theory in One and Higher Dimensions, Monographs and Textbooks in Pure and Applied Mathematics, 255, Marcel Dekker, Inc., New York, 2003.

[24] R.J. Libera, Some classes of regular univalent functions, Proc. Amer. Math. Soc., 16(1965), 755-758.

[25] P.T. Mocanu, Some simple criteria for starlikeness and convexity, Libertas Mathematica, 13(1993), 27-40.

[26] P.T. Mocanu, T. Bulboaca, Gr.S. Salagean, Geometric Function Theory of Univalent Functions(in Romanian), Casa Cartii de Stiinta, Cluj, 1999.

[27] G. Ren, X. Wang, Carathéodory theorems for slice regular functions, preprint 2014.

[28] M.I.S. Robertson, On the theory of univalent functions, Ann. of Math., (2) 37(1936), No. 2, 374-408. 
University of Oradea, Department of Mathematics and Computer Science, Str. Universitati Nr. 1, 410087 Oradea, Romania

E-mail address: galso@uoradea.ro

Departamento de Matemáticas, E.S.F.M. Del I.P.N. 07338, México D.F., México

E-mail address: jogc200678@gmail.com

Dipartimento di Matematica, Politecnico di Milano, Via Bonardi, 9, 20133 Milano, Italy

E-mail address: irene.sabadini@polimi.it 